\title{
BRaf signaling principles unveiled by large- scale human mutation analysis with a rapid lentivirus-based gene replacement method
}

\begin{abstract}
Chae-Seok Lim, ${ }^{1,2}$ Xi Kang, ${ }^{1}$ Vincent Mirabella, ${ }^{3,4}$ Huaye Zhang, ${ }^{3,5}$ Qian Bu, ${ }^{6,7}$ Yoichi Araki, ${ }^{8}$ Elizabeth T. Hoang, ${ }^{1,9}$ Shiqiang Wang, ${ }^{10}$ Ying Shen, ${ }^{11}$ Sukwoo Choi, ${ }^{2}$ Bong-Kiun Kaang, ${ }^{2}$ Qiang Chang, ${ }^{6,7}$ Zhiping P. Pang, ${ }^{3,4}$ Richard L. Huganir, ${ }^{8}$ and J. Julius Zhu ${ }^{1,12}$

${ }^{1}$ Department of Pharmacology, University of Virginia School of Medicine, Charlottesville, Virginia 22908, USA; ${ }^{2}$ Department of Biological Sciences, Seoul National University, Seoul 08826, Korea; ${ }^{3}$ Department of Neuroscience and Cell Biology, Rutgers Robert Wood Johnson Medical School, Piscataway, New Jersey 08854, USA; ${ }^{4}$ Child Health Institute of New Jersey, Rutgers Robert Wood Johnson Medical School, Piscataway, New Jersey 08854, USA; ${ }^{5}$ Department of Microbiology, Center for Cell Signaling, University of Virginia School of Medicine, Charlottesville, Virginia 22908, USA; ${ }^{6}$ Waisman Center, ${ }^{7}$ Department of Medical Genetics, University of Wisconsin School of Medicine, Madison, Wisconsin 53705, USA; ${ }^{8}$ Department of Neuroscience, Howard Hughes Medical Institute, Johns Hopkins University School of Medicine, Baltimore, Maryland 21205, USA; ${ }^{9}$ Undergraduate Class of 2014, Department of Psychology, University of Virginia College of Arts and Sciences, Charlottesville, Virginia 22908, USA; ${ }^{10}$ State Key Laboratory of Membrane Biology, School of Life Sciences, Peking University, Beijing 100871, China; ${ }^{11}$ Department of Neurobiology, Key Laboratory of Medical Neurobiology of Ministry of Health, Zhejiang University School of Medicine, Hangzhou 310058, China; ${ }^{12}$ Department of Neuroscience, University of Virginia School of Medicine, Charlottesville, Virginia 22908, USA
\end{abstract}

Rapid advances in genetics are linking mutations on genes to diseases at an exponential rate, yet characterizing the gene-mutation-cell-behavior relationships essential for precision medicine remains a daunting task. More than 350 mutations on $B R$ af are associated with various tumors, and $\sim 40$ mutations are associated with the neurodevelopmental disorder cardio-facio-cutaneous syndrome (CFC). We developed a fast cost-effective lentivirus-based rapid gene replacement method to interrogate the physiopathology of BRaf and $~ 50$ disease-linked BRaf mutants, including all CFC-linked mutants. Analysis of simultaneous multiple patch-clamp recordings from 6068 pairs of rat neurons with validation in additional mouse and human neurons and multiple learning tests from 1486 rats identified BRaf as the key missing signaling effector in the common synaptic NMDA-R-CaMKII-SynGap-RasBRaf-MEK-ERK transduction cascade. Moreover, the analysis creates the original big data unveiling three general features of BRaf signaling. This study establishes the first efficient procedure that permits large-scale functional analysis of human disease-linked mutations essential for precision medicine.

[Keywords: BRaf; cancer; CFC syndrome; neurodevelopmental disorder; synaptic transmission]

Supplemental material is available for this article.

Received November 29, 2016; revised version accepted March 13, 2017.

Precision medicine requires an understanding of gene-mutation-cell-behavior relationships on an individual basis. Rapid advances in genetics have identified large numbers of putative disease-linked mutations on numerous genes (Govek et al. 2005; Schubbert et al. 2007; Pavlowsky et al. 2012; Rauen 2013; Mei and Nave 2014; Siegert et al. 2015; Volk et al. 2015). However, how the disease-linked genes and mutations may differentially affect cellular functions and behavioral outputs remains poorly understood, primarily due to the time- and cost-prohibitive approaches for generating the large numbers of transgenic animal models required to understand the physiopathology of genes and their mutations (Govek et al. 2005; Siegert

Corresponding author: jizhu@virginia.edu

Article is online at http://www.genesdev.org/cgi/doi/10.1101/gad.294413. 116. et al. 2015; Volk et al. 2015). For example, geneticists have linked 40 mutations on the BRaf gene that encodes a serine/threonine protein kinase to cardio-facio-cutaneous syndrome (CFC) (Supplemental Fig. S1; Supplemental Table S1), a neurodevelopmental disorder with a $100 \%$ prevalence of learning impairment. CFC patients harboring these BRaf mutations exhibit a widely varied degree of cognitive impairment ranging from mild learning disability to profound mental retardation (Schubbert et al. 2007; Rauen 2013). What is still unclear is how the BRaf mutations lead to the different behavioral outcomes, which is

(C) 2017 Lim et al. This article is distributed exclusively by Cold Spring Harbor Laboratory Press for the first six months after the full-issue publication date (see http://genesdev.cshlp.org/site/misc/terms.xhtml). After six months, it is available under a Creative Commons License (Attribution-NonCommercial 4.0 International), as described at http://creativecommons.org/licenses/by-nc/4.0/. 
essential for developing personalized medicine for individual patients.

The sustained interest in Ras signaling stems from demonstrations of the essential role of Ras signaling in synaptic plasticity and identification of a large number of genetic mutations on various Ras signaling relaying and modulating molecules linked to mental, neurological, and psychiatric disorders (Costa and Silva 2003; Thomas and Huganir 2004; Stornetta and Zhu 2011). Accumulating evidence indicates that, in neuronal cells, activation of NMDA-sensitive glutamate receptors (NMDA-Rs) induces $\mathrm{Ca}^{2+}$ influx that can stimulate CaMKII-SynGapRas signaling, and downstream signaling of Ras is mediated by the MEK-ERK pathway that regulates AMPA-R-mediated synaptic transmission (English and Sweatt 1997; Zhu et al. 2002; Man et al. 2003; Kim et al. 2005; Qin et al. 2005; Lee et al. 2009; Patterson et al. 2010; Ozkan et al. 2014; Araki et al. 2015; Zeng et al. 2016). However, the signaling effector that links Ras to MEK-ERK at synapses remains elusive. In nonneuronal cells, canonical Ras signaling is relayed to the MEK-ERK pathway via the homodimerized CRaf (also called Raf-1) or heterodimerized CRaf and BRaf (Rauen 2013; Holderfield et al. 2014). Interestingly, we found previously that Ras signaling stimulated the activity of BRaf, but not CRaf, in CA1 neurons (Qin et al. 2005). This finding is supported by a knockout study that established a link between BRaf signaling and long-term potentiation (Chen et al. 2006) and is consistent with the earlier reports that BRaf was the sole Raf kinase expressed-and expressed at a high level—at synapses (Morice et al. 1999; Husi et al. 2000). These findings, which implicate a possible involvement of BRaf in canonical Ras signaling at synapses, underscore the importance of defining the precise synaptic function of BRaf (Nishiyama and Yasuda 2015; Volk et al. 2015).

We developed a fast cost-effective lentiviral tactic that allows for replacement of endogenous proteins with quantitatively and functionally equivalent amounts of recombinant mutant proteins, which is propitious for production of large numbers of animal models. Combining this method with high-throughput high-resolution multiple patch-clamp recordings and robust fear conditioning and Y-maze learning behavioral tests, we carried out a large-scale interrogation of the physiology and pathology of disease-linked BRaf and their mutations in rat, mouse, and human neurons. Our analysis identified BRaf to be the universal signaling effector relaying canonical NMDA-RCaMKII-SynGap-Ras signaling to the MEK-ERK cascade at synapses. The big data created in the analysis also unveiled three general principles governing BRaf signaling. First, loss-of-function and gain-of-function BRaf mutations induce a wide range of increases or decreases in AMPA-R-mediated transmission at synapses. Second, the level of BRaf mutation-induced alterations in transmission correlates with the degree of learning deficits in animals and the extent of cognitive impairments in human patients. Finally, all BRaf mutations impair its signaling, yet only those at sites crucial for interaction with ATP and substrate proteins can cause predominant deficits in BRaf signaling and cognition.

\section{Results}

To explore the synaptic function of BRaf, we first acutely overexpressed wild-type and mutant forms of BRaf in CA1 neurons in cultured rat hippocampal slices for $\sim 18$ $\mathrm{h}$ using the established Sindbis viral expression system (Fig. 1A; see Lim et al. 2014; Wang et al. 2015a for the method). Neurons expressing the GFP-tagged wild-type BRaf [GFP-BRaf(WT)] or a GFP-tagged constitutively active mutant BRaf(T599E/S602D) [GFP-BRaf(CA)] showed increased (by $\sim 30 \%$ ) AMPA-R-mediated responses compared with nearby control nonexpressing neurons. There was no difference in NMDA-R-mediated responses between GFP-BRaf(WT)-expressing or GFP-BRaf(CA)-expressing and control nonexpressing neurons (Fig. 1B,C; Supplemental Table S2), indicating a postsynaptic mechanism. To test whether endogenous BRaf activity potentiates transmission, we expressed a GFP-tagged dominantnegative mutant BRaf(K482M) [GFP-BRaf(DN)] or a GFPtagged kinase-impaired mutant BRaf(G469E) [GFP-BRaf (G469E)]. Neurons expressing GFP-BRaf(DN) or GFPBRaf(G469E) showed depressed (by $~ 30 \%$ ) AMPA responses compared with nearby control nonexpressing neurons (Fig. 1B,C; Supplemental Table S2), suggesting that blocking endogenous BRaf activity depressed synaptic transmission. These results suggest that endogenous BRaf activity contributes to a tonic potentiation of transmission. To determine whether activation of BRaf signaling requires synaptic activity, we included $12 \mathrm{mM} \mathrm{MgCl}$, which depresses synaptic transmission (Zhu et al. 2000), or APV, a pharmacological blocker of NMDA-Rs, in culture medium during expression of GFP-BRaf(WT) or GFP-BRaf(DN). High $\mathrm{Mg}^{2+}$ and APV blocked the effects of GFP-BRaf(WT) and GFP-BRaf(DN) on AMPA responses (Fig. 1C; Supplemental Table S2). These results suggest that the BRaf-mediated potentiation of synaptic transmission requires synaptic activity and activation of NMDA-Rs.

To determine whether BRaf may relay canonical CaMKII-SynGap-Ras signaling downstream to the MEK-ERK pathway at synapses, we cotransfected constitutively active tCaMKII (Zhu et al. 2002), SynGap(AA) (Araki et al. 2015), or Ras(CA) (Zhu et al. 2002; Qin et al. 2005) with BRaf(CA) or BRaf(DN) in rat hippocampal CA1 neurons in cultured slices (Fig. 2A). Neurons cotransfected with tCaMKII-GFP and RFP-BRaf(CA) and neurons cotransfected with RFP-Ras(CA) and GFP-BRaf(CA) showed $\sim 80 \%$ potentiations in AMPA responses compared with nearby control nontransfected neurons (Fig. 2B-E; Supplemental Table S3), the same as neurons expressing constitutively active tCaMKII or Ras alone (Zhu et al. 2002; Qin et al. 2005), suggesting an occlusion effect. In contrast, neurons cotransfected with tCaMKIIGFP and RFP-BRaf(DN) and neurons cotransfected with RFP-Ras(CA) and GFP-BRaf(DN) had reduced (by 30\%) AMPA responses (Fig. 2B-E; Supplemental Table S3), indicating a blockade of the tCaMKII-GFP-Ras(CA)-induced and RFP-Ras(CA)-induced synaptic potentiations by GFPBRaf(DN). CaMKII phosphorylates SynGap and drives synaptic removal of SynGap and thus up-regulates synaptic Ras signaling via a disinhibitory mechanism (Araki 
A A $\begin{gathered}\text { GFP-BRaf(WT), -BRaf(DN), } \\ \text {-BRaf (CA) or -BRaf(G469E) (Sindbis) }\end{gathered}$ (4) $\underset{\text { preparation }}{\text { Cultured slice }}$

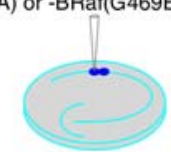

B Ctrl<smiles>CCCC(CC)CC</smiles>

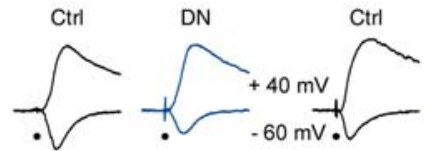

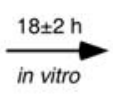

C
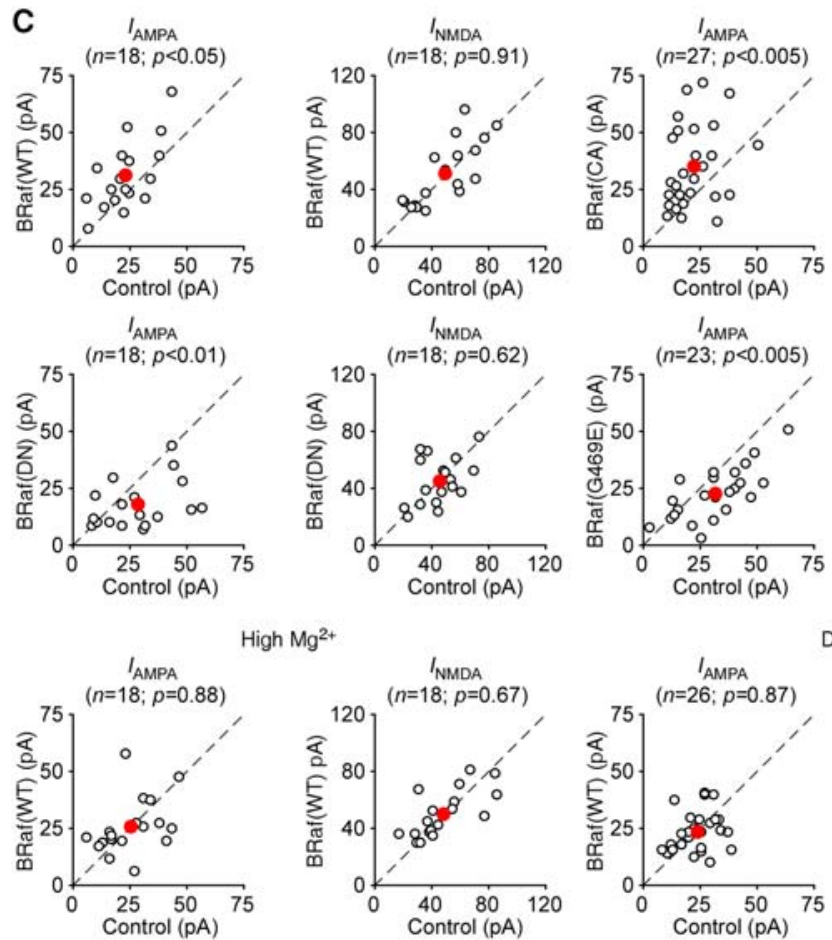

High $\mathrm{Mg}^{2+}$
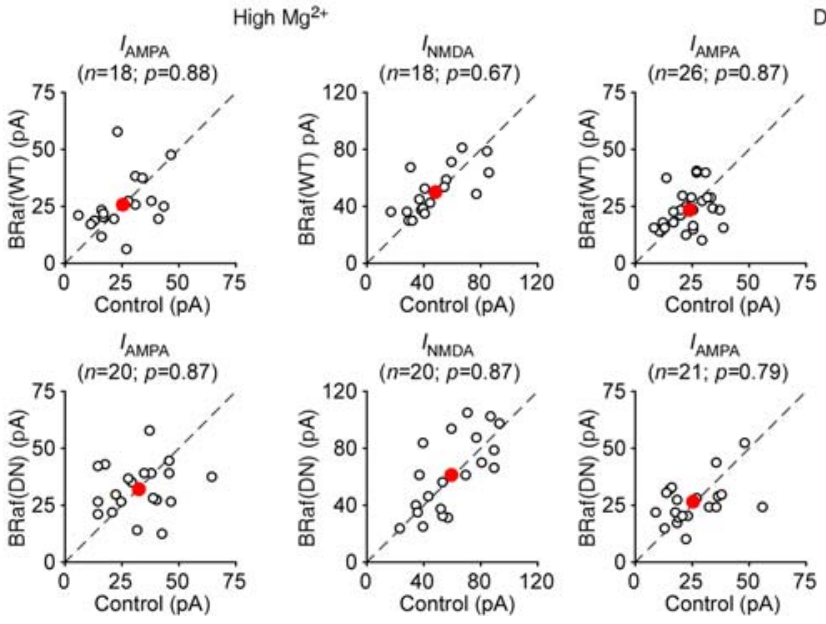

et al. 2015; Zeng et al. 2016). We found that neurons expressing phospho-deficient SynGap(AA)-GFP had reduced $(\sim 30 \%)$ AMPA responses compared with nearby control neurons and that neurons cotransfected with SynGap (AA)-GFP and RFP-BRaf(DN) showed reduced (by 30\%) AMPA responses (Fig. 2F, G; Supplemental Table S3), suggesting an occlusion effect. Moreover, neurons cotransfected with SynGap(AA)-GFP and RFP-BRaf(CA) had potentiated (by $\sim 50 \%$ ) AMPA responses (Fig. 2F, G; Supplemental Table S3), indicating a blockade of the SynGap(AA)-GFP-induced synaptic depression by RFP-BRaf (CA). No difference in NMDA responses was found in these experiments (Fig. 2; Supplemental Table S3). Together, these results indicate that BRaf is necessary and sufficient for relaying CaMKII, SynGap, and Ras signal(evoked EPSCs)
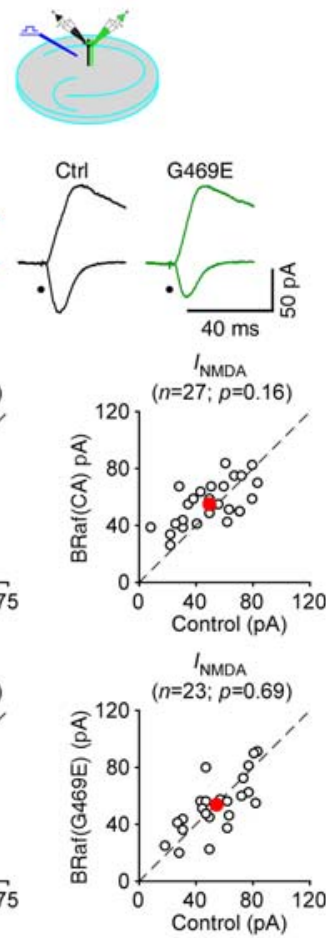

DL-APV
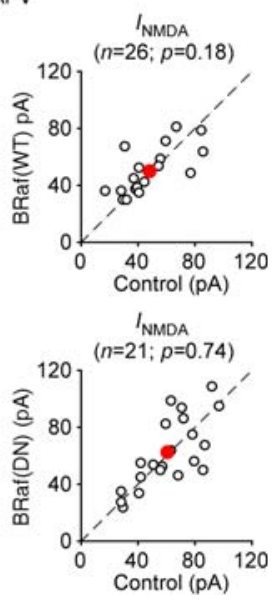

ing. We then investigated whether BRaf signals through the MEK-ERK pathway. Western blots showed increased phosphorylated p42/44 MAPK (also called ERK1/2) in CA1 cells of cultured rat hippocampal slices expressing GFP-BRaf(CA) and decreased phosphorylated p42/44 MAPK in CA1 cells of cultured rat hippocampal slices expressing GFP-BRaf(DN) (Supplemental Fig. S2A,B; Supplemental Table S4). Moreover, in the presence of PD98059 or U0126, both of which inhibit MEK, the p42/44 MAPK-activating enzyme, the GFP-BRaf(CA)-induced potentiation of AMPA responses was blocked, while the GFP-BRaf(DN)-induced depression of AMPA responses was occluded (Supplemental Fig. S2C; Supplemental Table S5). Collectively, these results support the existence of an NMDA-R-CaMKII-SynGap-Ras-BRaf- 
A

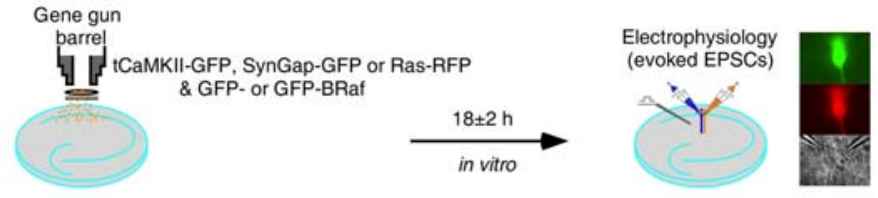

F Ctrl SynGap(AA)+BRaf(CA) Ctrl SynGap(AA)+BRaf(DN)
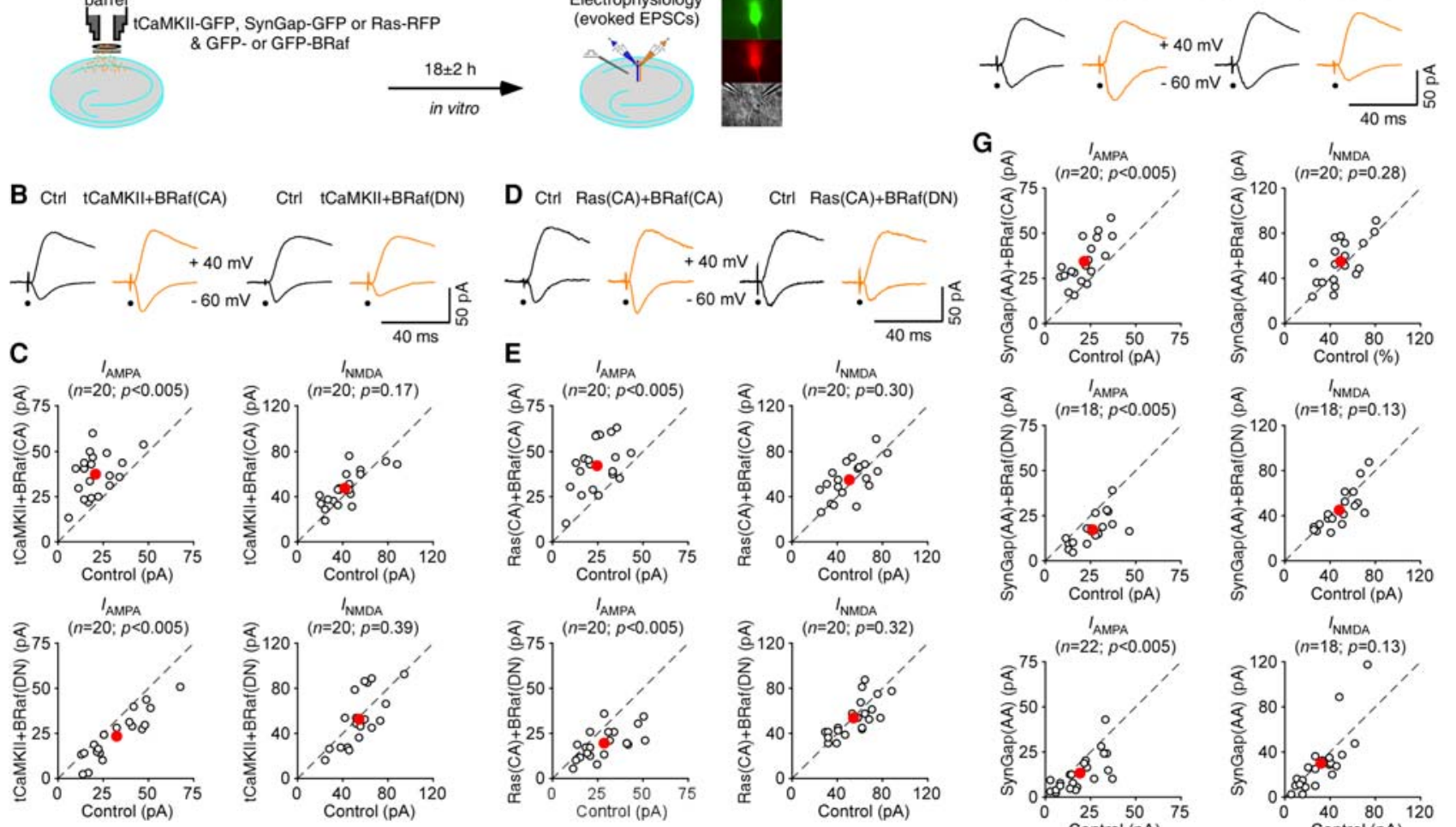
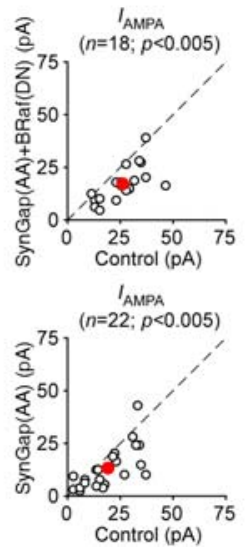

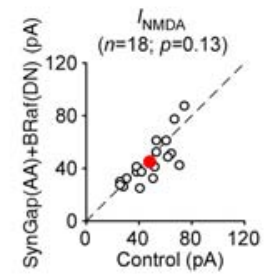

NMDA

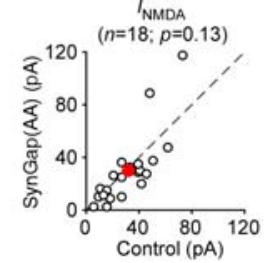

Figure 2. BRaf relays tCaMKII-SynGap-Ras signaling. (A) Schematic drawing outlining the design of coexpression experiments using cultured rat hippocampal slices. The images at the right show simultaneous whole-cell recordings from a pair of nonexpressing (Ctrl) and coexpressing CA1 neurons under transmitted light (bottom) and fluorescence microscopy with a GFP (top) or RFP (middle) filter. $(B)$ Evoked AMPA-R-mediated $(-60 \mathrm{mV})$ and NMDA-R-mediated $(+40 \mathrm{mV})$ responses recorded from nonexpressing neurons (Ctrl) and neurons coexpressing tCaMKII-GFP and RFP-BRaf(CA) or RFP-BRaf(DN). (C) AMPA and NMDA responses in neurons coexpressing tCaMKIIGFP and RFP-BRaf(CA) or RFP-BRaf(DN) are plotted against those obtained from neighboring control nonexpressing neurons. The large red dots with error bars indicate the averages and standard errors. See Supplemental Table S3 for values of AMPA and NMDA responses. $(D)$ Evoked AMPA-R-mediated $(-60 \mathrm{mV})$ and NMDA-R-mediated $(+40 \mathrm{mV})$ responses recorded from nonexpressing neurons (Ctrl) and neurons coexpressing RFP-Ras(CA) and GFP-BRaf(CA) or GFP-BRaf(DN). (E) AMPA and NMDA responses in neurons coexpressing RFP-Ras(CA) and GFP-BRaf(CA) or GFP-BRaf(DN) are plotted against those obtained from neighboring control nonexpressing neurons. The large red dots with error bars indicate the averages and standard errors. See Supplemental Table S3 for values of AMPA and NMDA responses. $(F)$ Evoked AMPA-R-mediated $(-60 \mathrm{mV})$ and NMDA-R-mediated $(+40 \mathrm{mV})$ responses recorded from nonexpressing neurons (Ctrl) and neurons coexpressing SynGap(AA)-GFP and RFP-BRaf(CA) or RFP-BRaf(DN). (G) AMPA and NMDA responses in neurons coexpressing SynGap(AA)-GFP and RFP-BRaf(CA) or RFP-BRaf(DN) are plotted against those obtained from neighboring control nonexpressing neurons. The large red dots with error bars indicate the averages and standard errors. See Supplemental Table S3 for values of AMPA and NMDA responses.

MEK-ERK signaling pathway that controls AMPA-R-mediated transmission at synapses.

\section{Development of a lentivirus-based rapid gene replacement method}

To further validate the physiological BRaf signaling at synapses and, more importantly, analyze the pathology of human disease-linked BRaf mutations, we developed a lentivirus-based replacement approach (Fig. 3A). Our lentiviral constructs used an $\mathrm{H} 1$ promoter to drive the shRNA-mediated knockdown of endogenous rat BRaf and an EF1 $\alpha$ promoter to express the replacement GFPtagged human BRaf (GFP-hBRaf). Because the EF1a promoter drove overexpression of GFP-hBRaf by 200\%$400 \%$, we inserted an internal ribosomal entry site
(IRES) sequence before GFP-hBRaf to reduce its expression and also express an additional copy of GFP (typically linked to a short membrane target sequence) (see below), which resulted in the total BRaf expression at similar levels of the endogenous protein $(\sim 90 \%-110 \%)$ during a 7 -d expression period (Fig. 3B). However, nonoverexpressed GFP-hBRaf and an additional copy of GFP did not provide sufficient fluorescence required for detecting expressing cells in light-scattering tissues, such as living brain slices. To identify expressing cells, we fused a short palmitoylation signal sequence of GAP43 to GFP (PalGFP), which concentrated PalGFP in the plasma membrane, forming conspicuous fluorescent rings encircling the somata of expressing neurons (Fig. 3C, inset).

We then examined whether the lentiviral construct could achieve the replacement with functionally 
A

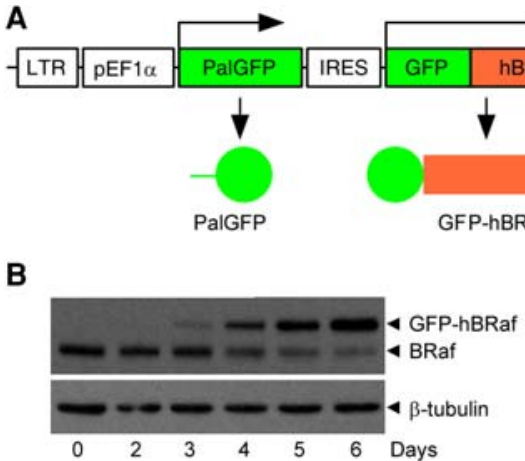

C

ShRNA-PalGFP-IRES-GFP-hBRaf(WT) ShRA-PalmCherry-IRES-mCherry-hBRaf(WT)
-shRNA-Palm

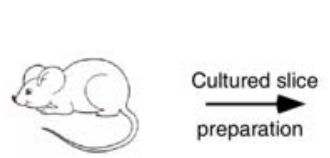
and/or ShRNA-PaICFP
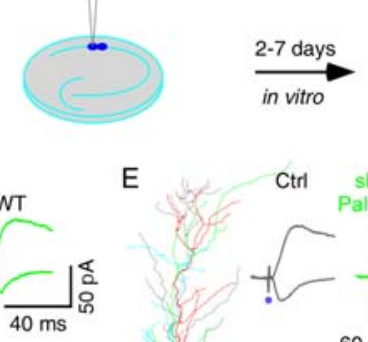

E

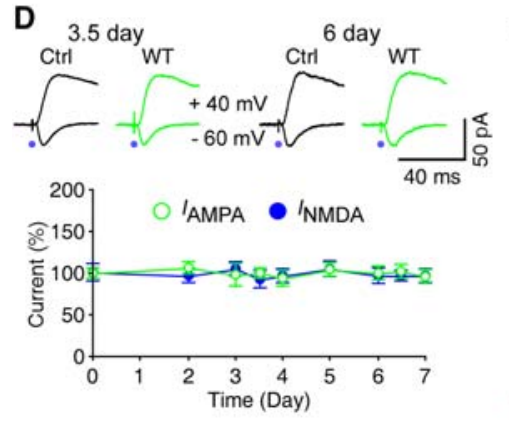

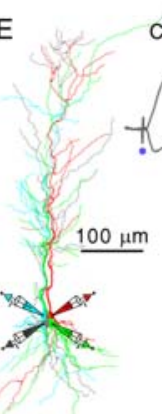

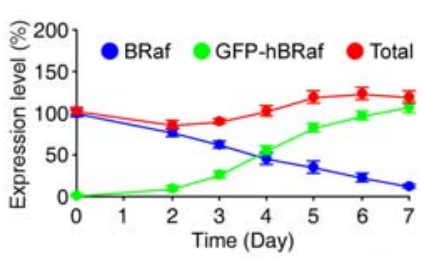

Time (Day) Electrophysiology (evoked EPSCs)
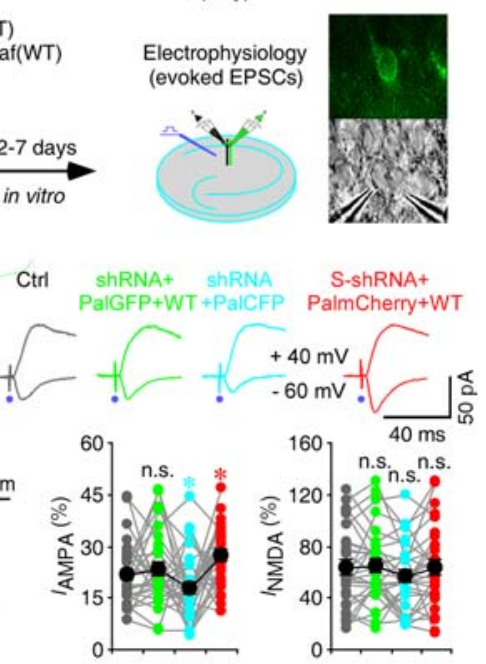

Figure 3. shRNA-PalGFP-IRES-GFP-hBRaf replaces rat BRaf with functionally equivalent hBRaf. (A) Design of a shRNAPalGFP-IRES-GFP-hBRaf construct. $(B$, left) Blots of endogenous rat BRaf, recombinant hBRaf, and $\beta$-tubulin in cultured rat hippocampal neurons after 2 to $6 \mathrm{~d}$ of lentiviral expression of shRNA-PalGFP-IRESGFP-hBRaf. (Right) Relative levels of endogenous BRaf and recombinant hBRaf after $2 \mathrm{~d}$ (BRaf, 76.3\% $\pm 4.6 \%$; hBRaf, 9.8\% $\pm 2.7 \%$; $n$ $=15$ from 15 animals), $3 \mathrm{~d}$ (BRaf, $61.6 \% \pm$ $4.1 \%$; hBRaf, $27.8 \% \pm 4.4 \% ; n=15$ from 15 animals), $4 \mathrm{~d}$ (BRaf, $46.6 \% \pm 6.6 \%$; hBRaf, $55.8 \% \pm 5.1 \% ; n=15$ from 15 animals), $5 \mathrm{~d}$ (BRaf, 35.3\% $\pm 7.3 \%$; hBRaf, $82.4 \% \pm 4.8 \%$; $n=15$ from 15 animals), $6 \mathrm{~d}$ (BRaf, $23.1 \% \pm$ $5.0 \%$; hBRaf, $98.8 \% \pm 5.0 \% ; n=15$ from 15 animals), and $7 \mathrm{~d}$ (BRaf, $12.3 \% \pm 3.6 \%$; hBRaf, $107.4 \% \pm 7.8 \% ; n=8$ from eight animals) of expression of shRNA-PalGFPIRES-GFP-hBRaf compared with control nonexpressing neurons (BRaf: $100.0 \% \pm$ $4.7 \%$; hBRaf: $2.6 \% \pm 1.1 \% ; n=15$ from 15 animals). The relative values and standard errors were normalized to average amounts of BRaf in control nonexpressing neurons. $(C)$ Schematic drawing outlining the design of in vitro experiments in cultured rat hippocampal slices. The inset fluorescence and transmitted light images show a simultaneously recorded expressing neuron, identified by the characteristic ring-like plasma membrane expression of PalGFP at its soma, and a neighboring control nonexpressing neuron. $(D$, top $)$ Evoked AMPA-R-mediated $(-60 \mathrm{mV})$ and NMDA-R-mediated $(+40 \mathrm{mV})$ responses recorded from neighboring nonexpressing (Ctrl) and shRNA-PalGFP-IRES-GFP-hBRaf(WT)-expressing cells after $3.5 \mathrm{~d}$ and $6 \mathrm{~d}$ of expression. (Bottom) Plots of amplitudes of synaptic AMPA and NMDA responses of expressing cells against expression time reveal no change in transmission after replacement of endogenous rat BRaf with recombinant hBR af for up to $7 \mathrm{~d}$. See Supplemental Table S6 for values of AMPA and NMDA responses. $(E, l e f t)$ Camera lucida reconstruction of control nonexpressing and expressing CA1 cells filled with biocytin. (Top right) Evoked AMPA-Rmediated $(-60 \mathrm{mV})$ and NMDA-R-mediated $(+40 \mathrm{mV})$ responses recorded from neighboring nonexpressing (Ctrl), shRNA-PalGFP-IRESGFP-hBRaf(WT)-expressing, shRNA-PalCFP-expressing, and scrambled shRNA (S-shRNA)-PalmCherry-IRES-mCherry-hBRaf(WT)-expressing cells after $3.5 \mathrm{~d}$ of expression. (Bottom right) Values for AMPA responses in shRNA-PalGFP-IRES-GFP-hBRaf(WT)-expressing, shRNA-PalCFP-expressing, and S-shRNA-PalmCherry-IRES-mCherry-hBRaf(WT)-expressing cells $(\mathrm{PalGFP},-23.2 \mathrm{pA} \pm 2.0 \mathrm{pA}, Z=$ $0.638, P=0.52$; PalCFP $,-17.4 \mathrm{pA} \pm 1.8 \mathrm{pA}, Z=-2.108, P<0.05$; PalmCherry, $-27.1 \mathrm{pA} \pm 1.7 \mathrm{pA}, Z=2.173, P<0.05)$ compared with control nonexpressing cells (Ctrl, $-21.8 \mathrm{pA} \pm 1.7 \mathrm{pA} ; n=29$ from 24 animals) and NMDA responses in shRNA-PalGFP-IRES-GFP-hBRaf(WT)-expressing, shRNA-PalCFP-expressing, and S-shRNA-PalmCherry-IRES-mCherry-hBRaf(WT)-expressing cells $(\mathrm{PalGFP}, 65.1 \mathrm{pA} \pm 5.4 \mathrm{pA}, Z$ $=0.497, P=0.62 ;$ PalCFP, $57.1 \mathrm{pA} \pm 4.9 \mathrm{pA}, Z=-1.330, P=0.18$; PalmCherry, $62.8 \mathrm{pA} \pm 6.0 \mathrm{pA}, Z=0.227, P=0.82)$ compared with control nonexpressing cells (Ctrl, $62.6 \mathrm{pA} \pm 5.6 \mathrm{pA} ; n=29$ from 24 animals). The large black dots with error bars indicate the averages and standard errors. $\left({ }^{*}\right) P<0.05$, Wilcoxon tests.

equivalent amounts of hBRaf(WT) by comparing synaptic transmission between expressing and nearby nonexpressing CA1 neurons in cultured rat hippocampal slices (Fig. 3C). During the 2- to 7-d expression period, shRNAPalGFP-IRES-GFP-hBRaf(WT)-expressing neurons had the same AMPA responses as nearby nonexpressing neurons (Fig. 3D; Supplemental Table S6), suggesting a functionally equivalent replacement. Moreover, neurons expressing shRNA-PalCFP, which had no replacement hBRaf(WT) sequence, had decreased AMPA responses, whereas neurons expressing a scrambled shRNA [SshRNA; S-shRNA-PalmCherry-IRES-mCherry-hBRaf
(WT)] had increased AMPA responses (Fig. 3E). There was no significant difference in NMDA responses between expressing and nonexpressing neurons (Fig. 3D,E). Finally, expression of shRNA-PalGFP-IRES-GFP-hBRaf had no effect on intrinsic membrane properties (i.e., the resting membrane potential, input resistance, and membrane time constant), paired pulse facilitation of AMPA responses, and GABA-R-mediated responses (Supplemental Fig. S3). Collectively, these results suggest that the lentiviral constructs make simultaneous knockdown of endogenous BRaf proteins and replacement with a quantitatively and functionally equivalent amount of recombinant hBRaf 
Lim et al.

proteins without causing any nonspecific effect on the basic membrane and synaptic properties.

\section{BRaf signals in neurons of various species}

We wished to know whether BRaf is a general signaling molecule that controls synaptic transmission in neurons. We first validated that BRaf signals synaptic transmission in CA1 neurons in cultured rat hippocampal slices by expressing the replacement constructs, including kinase-active mutants hBRaf(V600E) and hBRaf(K499E), kinaseimpaired mutant hBRaf(G469E), and dominant-negative mutant hBRaf(K482M). During the first 3.5-d expression period, shRNA-PalGFP-IRES-GFP-hBRaf(V600E)-expressing and shRNA-PalGFP-IRES-GFP-hBRaf(K499E)expressing rat CA1 neurons had increased AMPA responses, whereas shRNA-PalGFP-IRES-GFP-hBRaf(G469E)-ex- pressing and shRNA-PalGFP-IRES-GFP-hBRaf(K482M)expressing rat $\mathrm{CA} 1$ neurons had decreased AMPA responses (Fig. 4A,B; Supplemental Tables S7-S10). Interestingly, all expressing neurons had reduced AMPA responses at days 5-7 (Fig. 4A,B; Supplemental Tables S7-S10). There was no significant change in NMDA responses in all expressing neurons during the entire 7 -d expression period (Fig. 4A,B; Supplemental Tables S7-S10). These results confirm that BRaf replacement constructs up-regulate and down-regulate BRaf signaling to control AMPA-R-mediated synaptic transmission in rat CA1 neurons. We then repeated the experiments using CA1 neurons in cultured hippocampal slices prepared from mice (Supplemental Fig. S4A). As with rat CA1 neurons, after $3.5 \mathrm{~d}$ of expression, mouse CA1 neurons expressing shRNA-PalGFPIRES-GFP-hBRaf(V600E) and shRNA-PalGFP-IRES-GFPhBRaf(K499E) had increased AMPA responses, whereas
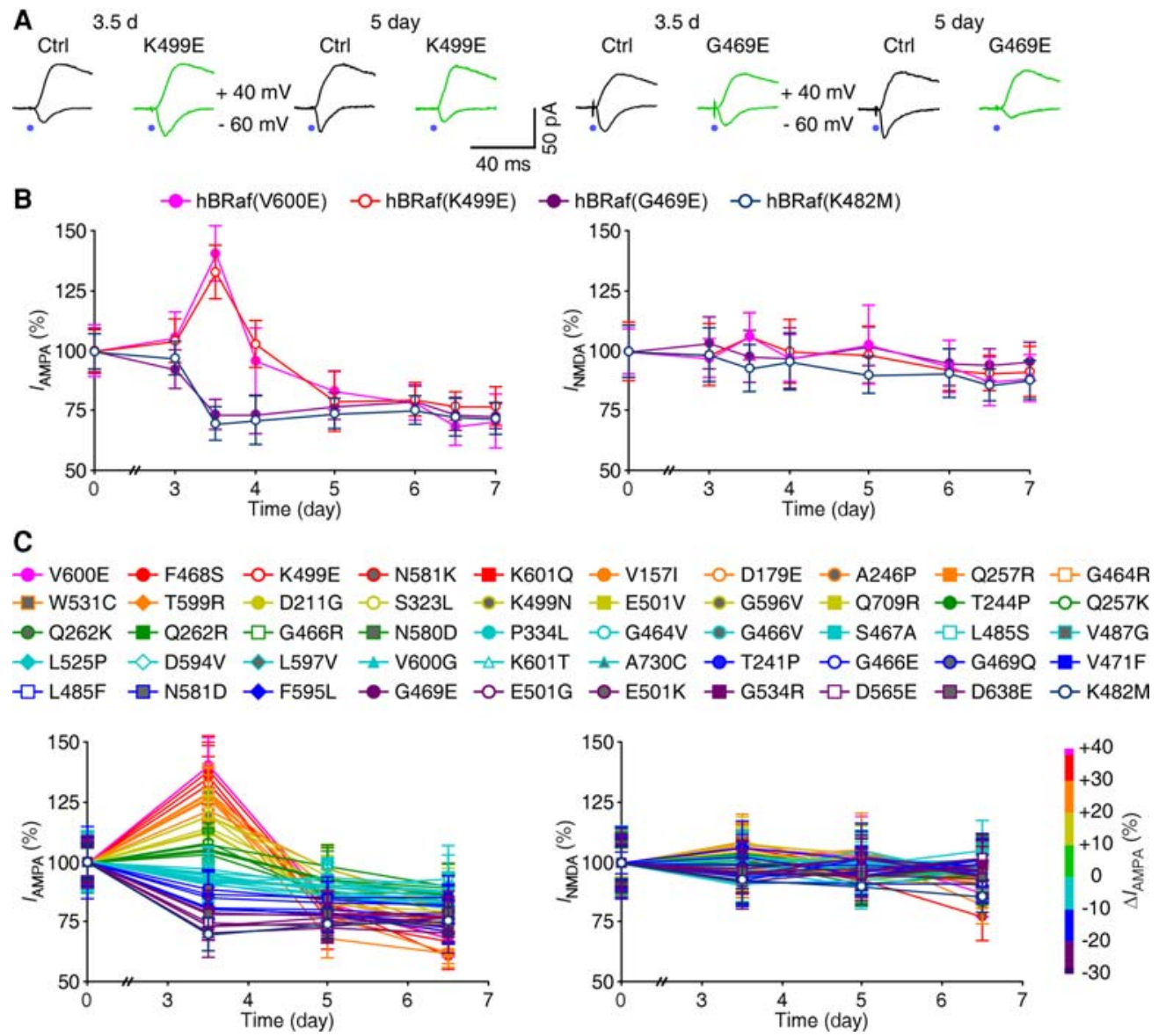

Figure 4. hBRaf mutants induce a wide range of changes in synaptic transmission. (A) Evoked AMPA-R-mediated $(-60 \mathrm{mV})$ and NMDAR-mediated $(+40 \mathrm{mV})$ responses recorded from neighboring nonexpressing (Ctrl) and shRNA-PalGFP-IRES-GFP-hBRaf(K499E)-expressing or shRNA-PalGFP-IRES-GFP-hBRaf(G469E)-expressing CA1 neurons in cultured rat hippocampal slices after $3.5 \mathrm{~d}$ and $5 \mathrm{~d}$ of expression. (B) Plots of amplitudes of synaptic AMPA and NMDA responses of cells expressing shRNA-PalGFP-IRES-GFP-hBRaf(V600E), shRNAPalGFP-IRES-GFP-hBRaf(K499E), shRNA-PalGFP-IRES-GFP-hBRaf(G469E), and shRNA-PalGFP-IRES-GFP-hBRaf(K482M) against expression time. See Supplemental Tables S7-S10 for values of AMPA and NMDA responses. (C) Plots of amplitudes of synaptic AMPA and NMDA responses of rat CA1 neurons expressing 50 disease-linked hBRaf mutant replacement constructs against expression time. See Supplemental Tables S15-S17 for values of AMPA and NMDA responses. AMPA-R-mediated and NMDA-R-mediated current amplitudes and standard errors were normalized to average values from control neurons. Note the mutants grouped and color-coded based on their effects on AMPA responses at day 3.5. 
those expressing shRNA-PalGFP-IRES-GFP-hBRaf(G469E) and shRNA-PalGFP-IRES-GFP-hBRaf(K482M) had decreased AMPA responses (Supplemental Fig. S4B,C; Supplemental Table S11). Moreover, mouse CA1 cells expressing shRNA-PalGFP-IRES-GFP-hBRaf(V600E) and shRNA-PalGFP-IRES-GFP-hBRaf(K499E) had increased levels of phosphorylated p42/44 MAPK, while those expressing shRNA-PalGFP-IRES-GFP-hBRaf(G469E) and shRNA-PalGFP-IRES-GFP-hBRaf(K482M) had decreased levels of phosphorylated p42/44 MAPK (Supplemental Fig. S4D,E; Supplemental Table S12). Together, these results suggest that BRaf signaling controls AMPA-R-mediated synaptic transmission via the MEK-ERK pathway in rodent neurons.

Given the very large number of BRaf mutations associated with human diseases, we wanted to know whether the finding may be extended to human neurons. Hence, we used human embryonic stem cells (ESCs) to induce human neuron cultures, which formed a functional neuronal network after $\sim 3-5$ wk in culture (Supplemental Fig. S5A,B; cf. Yi et al. 2016). We found that, in human ESC (hESC)-induced neurons, expression of shRNA-PalGFP-IRESGFP-hBRaf(V600E) and shRNA-PalGFP-IRES-GFP-hBRaf (K499E) increased the levels of phosphorylated p42/44 MAPK, whereas expression of shRNA-PalGFP-IRESGFP-hBRaf(G469E) and shRNA-PalGFP-IRES-GFP-hBRaf (K482M) decreased the levels of phosphorylated p42/44 MAPK (Supplemental Fig. S5C,D; Supplemental Table S13). Moreover, we generated induced pluripotent stem cell (iPSC)-derived neurons with human GM-1 cells. In human iPSC-derived neurons, expression of shRNA-PalGFPIRES-GFP-hBRaf(V600E) and shRNA-PalGFP-IRES-GFPhBRaf(K499E) increased the levels of phosphorylated p42/44 MAPK, whereas expression of shRNA-PalGFPIRES-GFP-hBRaf(G469E) and shRNA-PalGFP-IRES-GFPhBRaf(K482M) decreased the levels of phosphorylated p42/44 MAPK (Supplemental Fig. S6A-C; Supplemental Table S14). Collectively, these data establish the notion that BRaf is a general effector linking Ras signaling to the MEK-ERK pathway at synapses.

\section{Interrogation of the pathology of disease-linked human} BRaf mutants

Next, we systematically analyzed $\sim 50$ disease-linked hBRaf mutants (including all 40 CFC-linked mutants) in rat CA1 neurons. Expression of these hBRaf mutants for $3.5 \mathrm{~d}$ had either increased or decreased AMPA responses, resulting in a wide range of changes in synaptic AMPA responses, and, by days 5-7, all expressing neurons had depressed AMPA responses compared with nearby nonexpressing neurons (Fig. 4C; Supplemental Tables S15-S17). The majority of mutant hBRaf proteins had no significant effect on NMDA responses (Fig. 4C; Supplemental Tables S15-S17). There were significant correlations between the depressions or potentiations at day 3.5 and depression at days 5-7 for hBRaf mutants (Supplemental Fig. S7), suggesting the later depressions to be secondary to the initial potentiations. Both the potentiations and depressions were blocked during the period when the MEK inhibitor
PD98059 was included in culture medium (Supplemental Fig. S8; Supplemental Tables S18-S21), indicating the involvement of MEK-ERK signaling in the potentiations and depressions. Finally, the initial potentiations were prolonged, and the secondary depressions were delayed during the period when an IGFBP7 antibody, a-BP7, was included in culture medium (Supplemental Fig. S9; Supplemental Tables S22, S23), suggesting that the sustained Ras signaling-induced autocrine secretion of protein IGFBP7, which can stimulate apoptosis signaling (Wajapeyee et al. 2008) to depress AMPA responses (Li et al. 2010), may be responsible for the secondary depressions.

To examine whether BRaf controls synaptic transmission in intact brains, we combined an in vivo viral delivery technique and an acute slice preparation (McCormack et al. 2006; Lim et al. 2014). Specifically, we expressed shRNA-PalGFP-IRES-GFP-hBRaf mutant constructs in principal neurons in the lateral amygdala (LA) of intact rats for $3.5 \mathrm{~d}$. Subsequently, we made simultaneous recordings from paired control nonexpressing and expressing LA neurons in the acutely prepared amygdala slices (Fig. 5A). We compared synaptic responses evoked by electrical stimulation of LA-projecting fibers in the ventral striatum that originate from the auditory thalamus. LA neurons replaced with hBRaf(K499E) had enhanced AMPA responses, whereas LA neurons replaced with hBRaf(G469E) had reduced AMPA responses compared with nearby nonexpressing neurons (Fig. 5B,C; Supplemental Table S24). As a control, LA neurons replaced with hBRaf(WT) had the same AMPA responses (Fig. 5C; Supplemental Table S24). A systematic analysis revealed that replacement with the kinase active hBRaf(V600E), dominant-negative hBRaf(K482M), and 50 diseaselinked hBRaf mutants in rat LA neurons for $3.5 \mathrm{~d}$ had either increased or decreased AMPA responses, resulting in a wide range of changes in AMPA responses (Fig. 5C; Supplemental Table S24). There was no change in NMDA responses (Fig. 5C; Supplemental Table S24). Interestingly, the relative AMPA responses in BRaf mutant-expressing CA1 and LA neurons matched closely (Fig. 5C). These results indicate that hBRaf controls synaptic transmission both in vitro and in vivo.

To study whether BRaf signaling controls learning, we first investigated the effects of hBRaf signaling on learning behavior using the fear conditioning paradigm (Fig. 6A). We made bilateral massive lentiviral expression of shRNA-PalGFP-IRES-GFP-hBRaf mutant constructs in LAs of rats, which resulted in expression of $\sim 35 \%$ of LA neurons (Fig. 6A, inset). During the fear conditioning training, rats expressing hBRaf(K499E) and hBRaf(G469E) mutants had the same responses to a tone presentation and electrical foot shock and exhibited the same amount of freezing compared with control nonexpressing rats or rats expressing hBRaf(WT) (Fig. 6B). During the testing $24 \mathrm{~h}$ after fear conditioning, rats expressing hBRaf (K499E) or hBRaf(G469E) mutants displayed reduced amounts of freezing in both contextual and cued fear conditioning tests (Fig. 6B), indicating impairments of contextual and cued fear memory by hBRaf(K499E) and hBRaf(G469E) mutants. As a control, rats expressing 
A

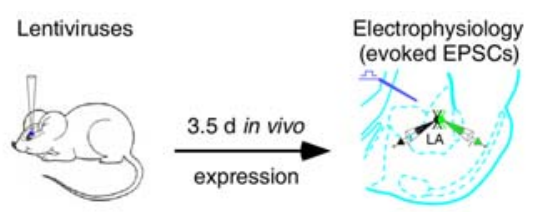

B

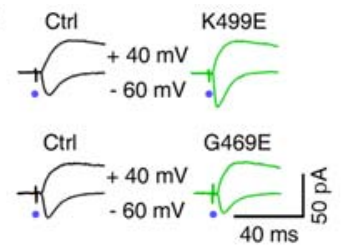

C

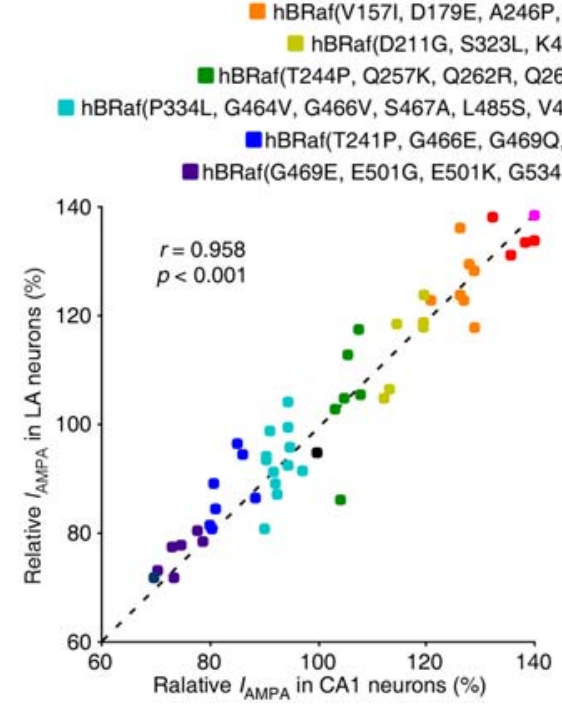

nBRaf(V600E) hiRRaf(F468S, K499E, N581K \& K601Q)

inRRaf(D211G, S323L, K499N, E501V, G596V \& Q709R)

anBRaf(WT)

nBRaf(T241P, G466E, G469Q, V471F, L485F, N581D \& F595L)

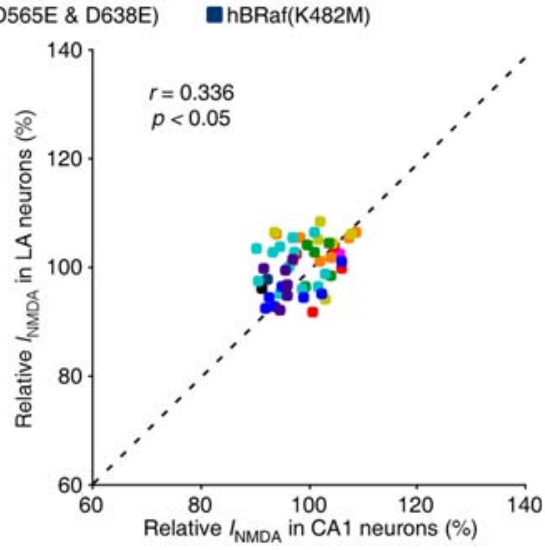

Figure 5. hBRaf mutants induce a wide range of changes in synaptic transmission in vivo. (A) Schematic drawing outlining the design of experiments using in vivo viral expression and in vitro acute rat LA slice preparation. $(B)$ Evoked AMPA-R-mediated $(-60 \mathrm{mV})$ and NMDA-R-mediated $(+40 \mathrm{mV})$ responses recorded from neighboring nonexpressing (Ctrl) and shRNA-PalGFP-IRESGFP-hBRaf(K499E)-expressing or shRNAPalGFP-IRES-GFP-hBRaf(G469E)-expressing LA neurons in acutely prepared LA slices after $3.5 \mathrm{~d}$ of in vivo expression. (C) Plots show strong correlation between the relative amplitudes of synaptic AMPA responses $(n$ $=51$; normality test, $P=0.80$; constant variance, $P=0.28 ; r=0.958, P<0.001$ ) but weak correlation between the relative amplitudes of synaptic NMDA responses $(n=46$; normality test, $P=0.25$; constant variance, $P=$ $0.40 ; r=0.336, P<0.05$; linear regression, $t$ test) of hBRaf mutant-expressing LA neurons and CA1 neurons. See Supplemental Table S24 for values of AMPA and NMDA responses of LA neurons expressing $\sim 50$ disease-linked hBRaf mutant replacement constructs. AMPA-R-mediated and NMDA-Rmediated current amplitudes and standard errors were normalized to average values from control neurons.
hBRaf(WT) exhibited the same amount of freezing compared with nonexpressing rats (Fig. 6B). Expressing constitutively active hBRaf(V600E), dominant-negative hBRaf(K482M), and 50 disease-linked hBRaf mutants showed a wide range of changes in the amount of freezing in both contextual and cued fear conditioning tests, and the amount of freezing in expressing animals correlated with the altered AMPA responses in expressing LA neurons (Fig. 6B; Supplemental Table S25).

To determine how the loss and gain of function of hBRaf mutations led to learning defects, we examined synaptic responses in paired control nonexpressing and control expressing LA neurons in the amygdala slices acutely prepared after fear conditioning (Supplemental Fig. S10A). We found that LA neurons expressing hBRaf(G469E) or hBRaf(K482M) had more reduced AMPA responses compared with nearby nonexpressing neurons (Supplemental Fig. S10B,C; Supplemental Table S26), suggesting a blockade of the fear conditioning-induced synaptic potentiation by the loss of function of hBRaf mutations /cf. Takahashi et al. 2003; McCormack et al. 2006; Kielland et al. 2009). Moreover, LA neurons expressing hBRaf(K499E) or hBRaf (V600E) had the same AMPA responses compared with nearby nonexpressing neurons (Supplemental Fig. S10B, C; Supplemental Table S26), suggesting an occlusion of the fear conditioning-induced synaptic potentiation by the gain of function of hBRaf mutations (cf. McCormack et al. 2006; Kielland et al. 2009). These results suggest that down-regulation and up-regulation of BRaf signaling block and occlude synaptic potentiation, respectively, both of which may thus reduce the capacity of synaptic plasticity critical for learning (cf. McCormack et al. 2006).

To validate the effects of hBRaf mutations on learning, we repeated the experiments using the elevated Y-maze learning task, a hippocampus-dependent learning para$\operatorname{digm}$ (Schmitt et al. 2005; Lim et al. 2014). We used the replacement constructs to express hBRaf(WT), hBRaf (K499E), and hBRaf(G469E) in both sides of the hippocampus (Fig. 7A). Multiple injections of high-titer viral solution in the hippocampi in both hemispheres resulted in $\sim 35 \%$ of CA1 neurons expressing replacement hBRaf (Fig. 7A, inset). Rats expressing hBRaf(K499E) or hBRaf (G469E) had impaired Y-maze learning compared with nonexpressing rats or rats expressing hBRaf(WT) (Fig. 7B). The control experiments showed that rats expressing hBRaf(WT) learned the Y-maze task as efficiently as nonexpressing rats (Fig. 7B). A full analysis of the kinase-active hBRaf(V600E), dominant-negative hBRaf(K482M), and $\sim 50$ disease-linked hBRaf mutants revealed that learning impairments in hBRaf-expressing animals correlated with the amounts of change in AMPA responses in hBRaf-expressing CA1 neurons (Fig. 7B; Supplemental Table S27). Together, these results validate that BRaf signaling controls learning behavior.

Cognitive impairment of human subjects has been examined in only a small population of CFC patients due in part to the difficulty in patient recruitment, and, frequently, the clinical studies were only able to link BRaf mutations to rough measurements of cognitive impairment (i.e., mild, moderate, severe, or profound mental 
A
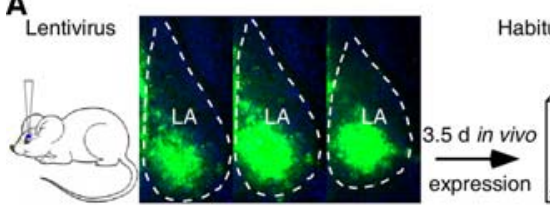

Habituation \& conditioning
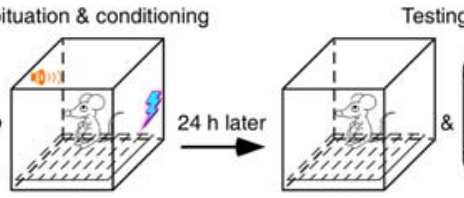

Testing

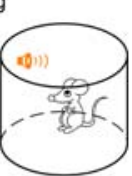

B

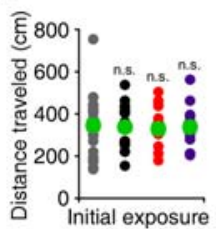

- Control
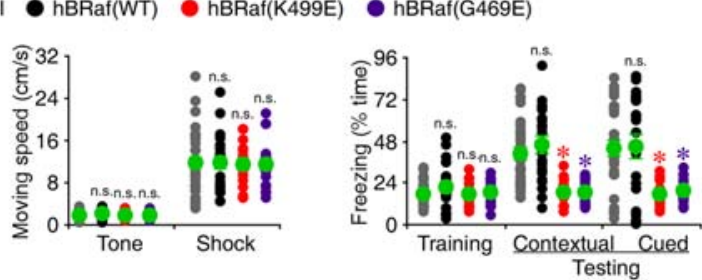

C

WhBRaf(V600E) $\quad$ hBRaf(F468S, K499E, N581K \& K601Q)

= $\mathrm{hBRaf}(\mathrm{V} 1571, \mathrm{D} 179 \mathrm{E}, \mathrm{A} 246 \mathrm{P}, \mathrm{Q} 257 \mathrm{R}, \mathrm{G} 464 \mathrm{R}$, W531C \& T599R) hBRaf(D211G, S323L, K499N, E501V, G596V \& Q709R) -hBRaf(T244P, Q257K, Q262R, Q266K, G466R \& N580D) m nBRaf(WT)

hBRaf(P334L, G464V, G466V, S467A, L485S, V487G, L525P, D594V, L597V, V600G, K601T \& A730C)

mBRaf(T241P, G466E, G469Q, V471F, L485F, N581D \& F595L)

mBRaf(G469E, E501G, E501K, G534R, D565E \& D638E) -hBRaf(K482M)
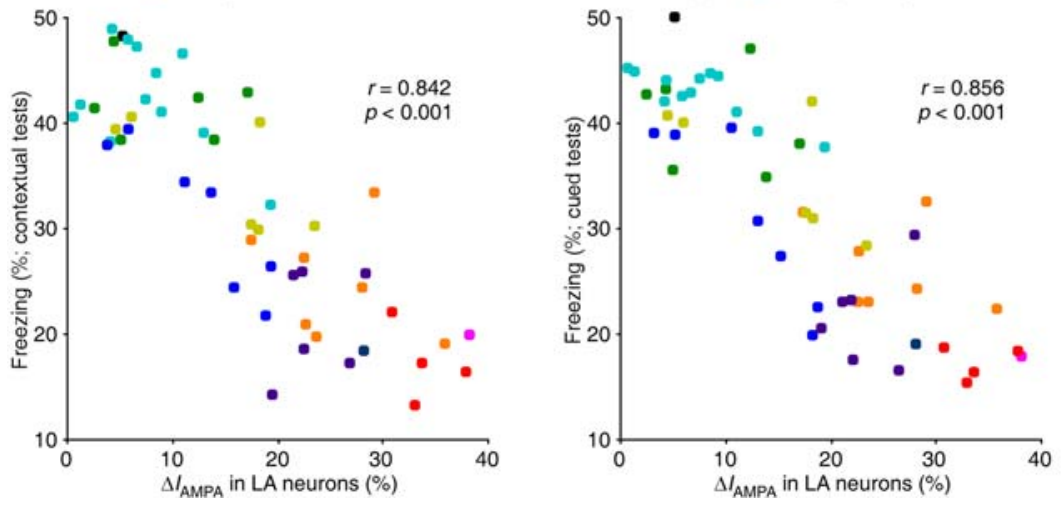

Figure 6. hBRaf mutants induce varied degrees of learning deficits in fear conditioning tasks. (A) Schematic drawing outlining the design of fear conditioning tasks for rats. The inset fluorescence images show the massive expression of shRNA-PalGFPIRES-GFP-hBRaf in the LA. (B, left) Distances traveled during the initial exposure for animals expressing shRNA-PalGFPIRES-GFP-hBRaf(WT), shRNA-PalGFPIRES-GFP-hBRaf(K499E), or shRNAPalGFP-IRES-GFP-hBRaf(G469E) [hBRaf (WT), $331.7 \mathrm{~cm} \pm 22.3 \mathrm{~cm}, n=20, U=300.5$, $P=0.84 ; \quad$ hBRaf(K499E), $328.0 \quad \mathrm{~cm} \pm 25.1$ $\mathrm{cm}, \quad n=14, \quad U=221.0, \quad P=0.65 ; \quad$ hBRaf (G469E), $319.3 \mathrm{~cm} \pm 27.8 \mathrm{~cm}, \quad n=14$, $U=228.5, P=0.52]$ compared with control nonexpressing animals (Ctrl, $340.4 \mathrm{~cm} \pm$ $24.0 \mathrm{~cm}, n=29$ ). (Middle) Moving speeds for animals expressing shRNA-PalGFPIRES-GFP-hBRaf(WT), shRNA-PalGFPIRES-GFP-hBRaf(K499E), or shRNAPalGFP-IRES-GFP-hBRaf(G469E) during the tone presentation [hBRaf(WT), 1.86 $\mathrm{cm} / \mathrm{sec} \pm 0.19 \mathrm{~cm} / \mathrm{sec}, n=20, U=258.0, P=$ 0.52; hBRaf(K499E), $1.83 \mathrm{~cm} / \mathrm{sec} \pm 0.23$ $\mathrm{cm} / \mathrm{sec}, n=14, U=191.0, P=0.77$; hBRaf (G469E), $1.69 \mathrm{~cm} / \mathrm{sec} \pm 0.18 \mathrm{~cm} / \mathrm{sec}, n=14$, $U=206.0, P=0.95]$ compared with control nonexpressing animals (Ctrl, $1.73 \mathrm{~cm} / \mathrm{sec} \pm$ $0.16 \mathrm{~cm} / \mathrm{sec}, n=29$ ) and those during electric foot shock [hBRaf(WT), $11.6 \mathrm{~cm} / \mathrm{sec} \pm$ $1.3 \mathrm{~cm} / \mathrm{sec}, \quad n=20, \quad U=288.5, \quad P=0.98$; hBRaf(K499E), $12.2 \pm 1.3 \mathrm{~cm} / \mathrm{sec}, \quad n=14$, $U=182.0, P=0.60 ;$ hBRaf(G469E), $11.9 \mathrm{~cm} /$ $\mathrm{sec} \pm 1.3 \mathrm{~cm} / \mathrm{sec}, n=14, U=190.0, P=0.75]$ compared with control nonexpressing animals (Ctrl, $11.7 \mathrm{~cm} / \mathrm{sec} \pm 1.2 \mathrm{~cm} / \mathrm{sec}, n=$

29). (Right) Amounts of freezing for animals expressing shRNA-PalGFP-IRES-GFP-hBRaf(WT), shRNA-PalGFP-IRES-GFP-hBRaf (K499E), or shRNA-PalGFP-IRES-GFP-hBRaf(G469E) during training [hBRaf(WT), 21.7\% $\pm 2.8 \%, n=20, U=238.0, P=0.30 ; \mathrm{hBR}$ af (K499E), $20.0 \% \pm 2.3 \%, n=14, U=174.0, P=0.46$; hBRaf(G469E), $20.2 \% \pm 2.3 \%, n=14 ; U=162.5, P=0.30]$ compared with control nonexpressing animals (Ctrl, $17.5 \% \pm 1.3 \%, n=29)$; during a contextual fear conditioning test [hBRaf(WT), 48.1\% $\pm 4.6 \%, n=20 ; U=297.0, P=$ 0.90; hBRaf(K499E), $16.3 \% \pm 2.7 \%, n=14, U=372.0, P<0.001$; hBRaf(G469E), $18.5 \% \pm 2.7 \%, n=14, U=369.0, P<0.001]$ compared with control nonexpressing animals $(\mathrm{Ctrl}, 48.8 \% \pm 4.0 \%, n=29)$; and during cued fear conditioning tests $[\mathrm{hBRaf}(\mathrm{WT}), 50.1 \% \pm 5.5 \%, n=20, U=$ 278.5, $P=0.82$; hBRaf(K499E), $18.3 \% \pm 4.6 \%, n=14, U=344.0, P<0.001$; hBRaf(G469E), $17.5 \% \pm 2.8 \%, n=14, U=361.0, P<0.001]$ compared with control nonexpressing animals $(\mathrm{Ctrl}, 48.9 \% \pm 4.6 \%, n=29)$. The large green dots with error bars indicate the averages and standard errors. $\left({ }^{*}\right) P<0.05$, Mann-Whitney rank sum test. $(C)$ Plots show correlations between the amounts of freezing of hBRaf mutantexpressing animals in contextual $(n=51$; normality test, $P=0.60$; constant variance, $P=0.62 ; r=0.842, P<0.001)$ and cued $(n=51$; normality test, $P=0.85$; constant variance, $P=0.07 ; r=0.856, P<0.001$; linear regression $t$-test ) fear conditioning tests and altered synaptic AMPA responses of corresponding hBRaf mutant-expressing LA neurons. See Supplemental Table S25 for values of the amount of freezing of animals expressing $\sim 50$ disease-linked hBRaf mutant replacement constructs.

retardation) (Yoon et al. 2007; Demir et al. 2010; Pierpont et al. 2010; Alfieri et al. 2014). Nevertheless, while scrutinizing these clinical reports, we noted that CFC patients carrying the same BRaf mutations exhibit similar intellectual disabilities (Supplemental Table S28). Thus, we compared these clinical results with our animal data. The extent of cognitive impairment of CFC patients correlated well with the degree of alteration in AMPA responses in hBRaf mutant-expressing neurons in rats and the level of deficit in learning in hBRaf mutant-expressing animals
(Fig. 8A). These results suggest that the effects of CFClinked hBRaf mutations on synaptic transmission and learning predict the severity of cognitive impairment in human patients.

\section{Discussion}

In this study, we developed and validated a simple and rapid gene replacement method that allows us to carry out a 
A

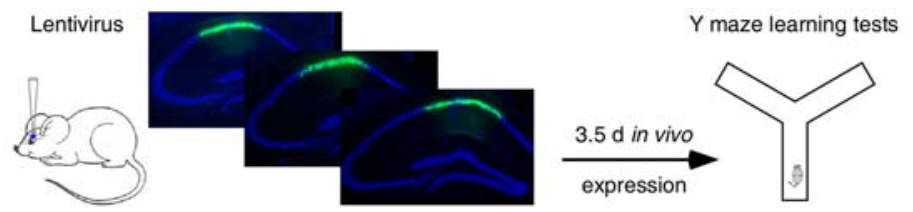

B

hBRaf(V600E) hBRaf(F468S, K499E, N581K \& K601Q)

nBRaf(V157I, D179E, A246P, Q257R, G464R, W531C \& T599R)

= hBRaf(D211G, S323L, K499N, E501V, G596V \& Q709R)

mBRaf(T244P, Q257K, Q262R, Q266K, G466R \& N580D) m hBRaf(WT)

= hBRaf(P334L, G464V, G466V, S467A, L485S, V487G, L525P, D594V, L597V, V600G, K601T \& A730C)

ahBRaf(T241P, G466E, G469Q, V471F, L485F, N581D \& F595L)

ahBRaf(G469E, E501G, E501K, G534R, D565E \& D638E) —hBRaf(K482M)
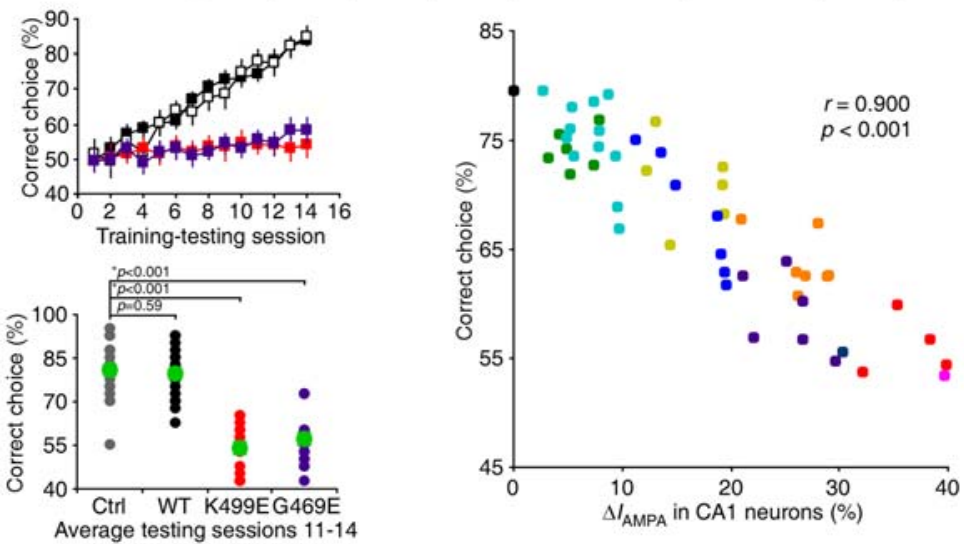

Figure 7. hBRaf mutants induce varied degrees of learning deficits in Y-maze tasks. (A) Schematic drawing outlining the design of Y-maze tasks for rats. The inset fluorescence images show the massive expression of shRNA-PalGFP-IRESGFP-hBRaf in the hippocampal CAl region. $(B$, top left) During the elevated Y-maze learning test, animals expressing shRNA-PalGFP-IRESGFP-hBRaf(K499E) or shRNA-PalGFP-IRES-GFPhBRaf(G469E) exhibited a significant reduction in the percentage of correct choices compared with control nonexpressing animals and animals expressing shRNA-PalGFP-IRES-GFP-hBRaf(WT). (Bottom left) The percentages of correct choices in animals expressing shRNA-PalGFP-IRES-GFP-hBRaf(WT) $(79.5 \% \pm 1.8 \%, n=20, U=177.0, P=$ 0.54), shRNA-PalGFP-IRES-GFP-hBRaf(K499E) $(53.6 \% \pm 2.1 \%, n=18, U=350.5, P<0.001)$, or shRNA-PalGFP-IRES-GFP-hBRaf(G469E) (56.7\% $\pm 2.1 \%, \quad n=18, \quad U=343.0, \quad P<0.001$ ) compared with control nonexpressing animals $180.6 \% \pm$ $2.1 \%, n=20$ ). The large green dots with error bars indicate the averages and standard errors. (*) $P<0.05$, Mann-Whitney rank sum tests. (Right) The plot shows correlation between correct choices of hBRaf mutant-expressing animals and altered synaptic AMPA responses of corresponding hBRaf mutant-expressing CA1 neurons ( $n=51$; normality test, $P=0.51$; constant variance, $P=0.12 ; r=0.900, P<0.001$; linear regression $t$-test). See Supplemental Table S27 for values of the correct choices of animals expressing $\sim 50$ disease-linked hBRaf mutant replacement constructs. large-scale analysis of physiological and pathological BRaf signaling in vitro and in intact brains. The analysis creates more than one order of magnitude of more data that unveil a general role of BRaf in relaying canonical Ras signaling to the MEK-ERK pathway in neurons of multiple species (i.e., rats, mice, and humans); the data identify BRaf as the key missing relay effector, and thus this study completes the synaptic NMDA-R-CaMKII-SynGap-RasBRaf-MEK-ERK signal transduction cascade that controls synaptic AMPA-R-mediated transmission (Fig. 8B). Moreover, the big data permit one to decipher the general principles that govern BRaf signaling (Fig. 8B), opening cell signaling research to a new dimension. Particularly, our analysis of $\sim 50$ BRaf mutations reveals that CFC- and/or cancer-linked BRaf mutants induce either an increase or a decrease in signaling that leads to a wide range of changes in synaptic transmission and learning impairment. While mutations in various BRaf domains all cause alterations in BRaf signaling, at several sites crucial for interaction with ATP and substrate proteins, they can lead to predominant BRaf signaling deficits. The degree of BRaf mutation-induced alterations in transmission and deficits in learning in animals correlate with the extent of cognitive impairment seen in CFC patients, which ranges from a mild learning disability to profound mental retardation.

The Ras family GTPases (i.e., Ras, Rap1, and Rap2) independently control distinct forms of synaptic plasticity via their downstream protein kinase signaling cascades
(Costa and Silva 2003; Thomas and Huganir 2004; Stornetta and Zhu 2011). Specifically, the Ras-MEK-ERK and Ras-PI3K-AKT pathways signal phosphorylation and synaptic delivery of AMPA-Rs containing subunits with long cytoplasmic termini during long-term potentiation, and the Rap1-p38 MAPK pathway signals phosphorylation and synaptic removal of AMPA-Rs containing subunits with only short cytoplasmic termini during long-term depression (Zhu et al. 2002; Qin et al. 2005; McCormack et al. 2006; Kielland et al. 2009), whereas the Rap2-JNK-PP2B pathway signals dephosphorylation and synaptic removal of AMPA-Rs containing subunits with long cytoplasmic termini during depotentiation (Zhu et al. 2005; Sheng et al. 2016). The canonical Ras-MEKERK pathway is one of the best-studied signaling pathways because of its involvement in several key cellular processes, including cell proliferation, differentiation, survival and cell motility, and because of its potential in being a drug target (Samatar and Poulikakos 2014; Caunt et al. 2015). In neuronal cells, the Ras-MEK-ERK pathway also controls synaptic transmission and plasticity. Accumulating data have linked synaptic NMDA-R activation to $\mathrm{Ca}^{2+}$ influx, then CaMKII-SynGap-Ras signaling, and finally downstream MEK-ERK signaling that controls synaptic AMPA-R phosphorylation and trafficking (Zhu et al. 2002; Man et al. 2003; Kim et al. 2005; Qin et al. 2005; Yasuda et al. 2006; Lee et al. 2009; Patterson et al. 2010; Araki et al. 2015; Zeng et al. 2016). However, how Ras signaling is relayed to the MEK-ERK pathway at 

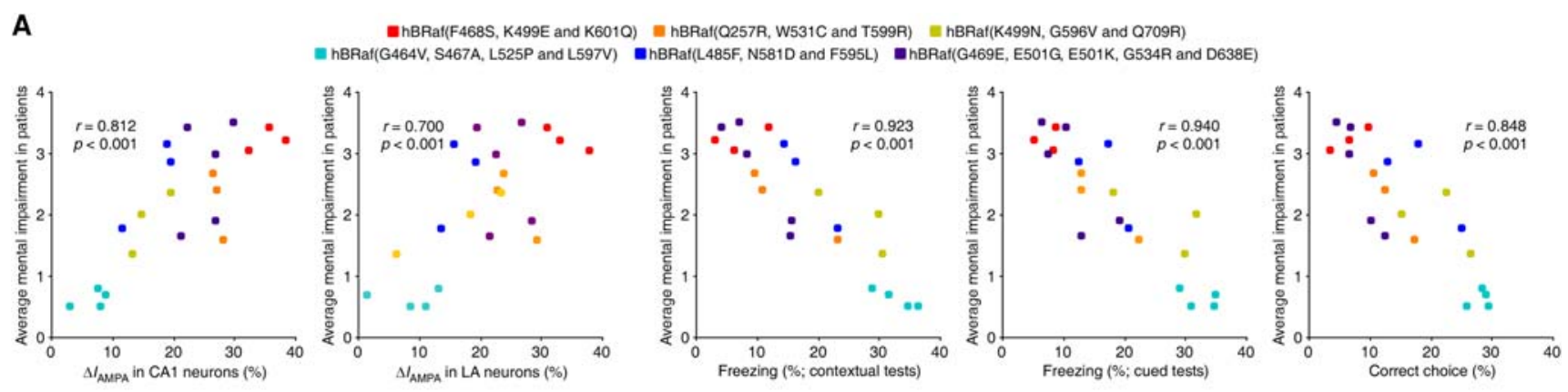

B

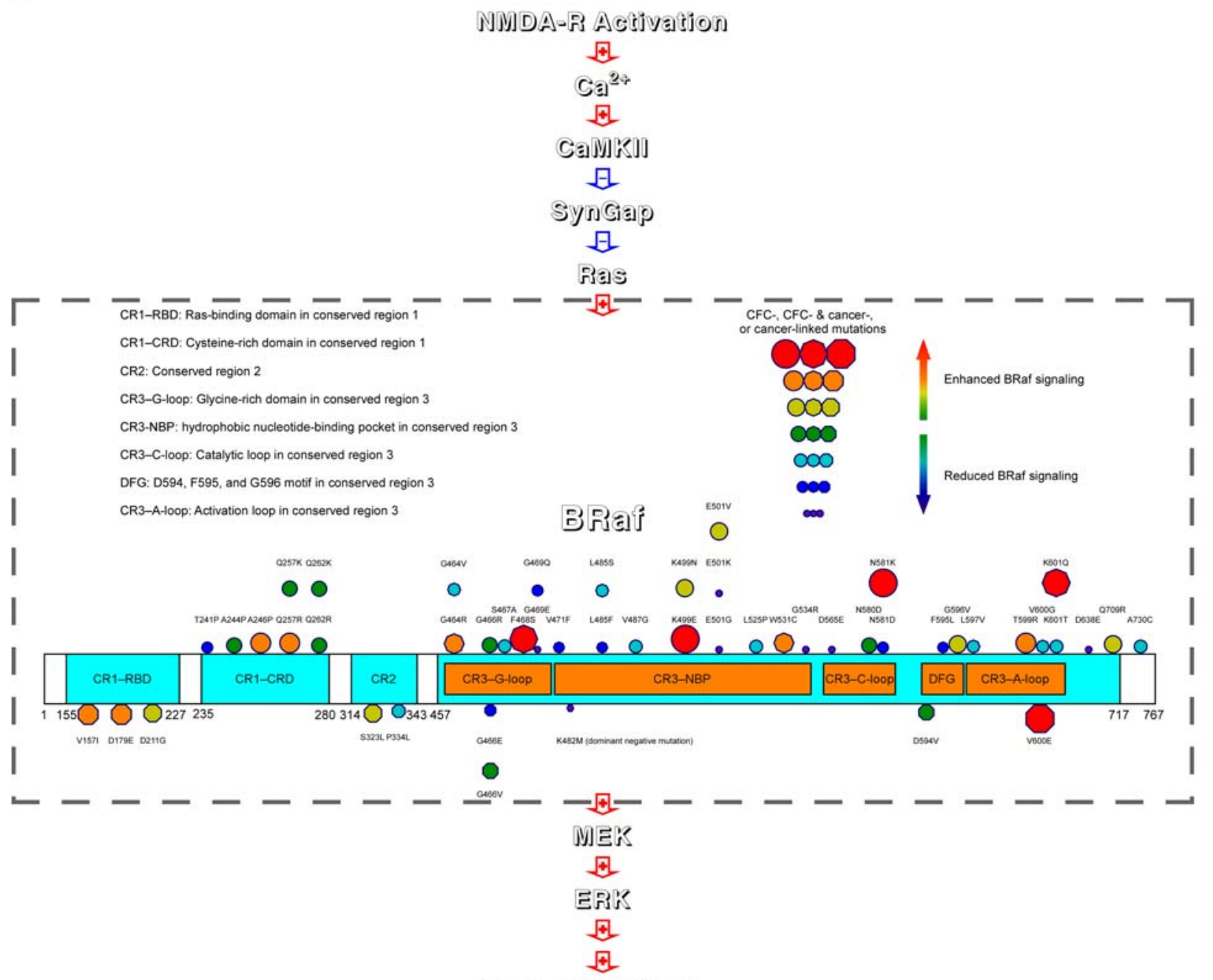

AMPA-R Trafificking

Figure 8. hBRaf mutants predict cognitive impairments in CFC patients. $(A)$ The two plots at the left show correlations between cognitive impairments of CFC patients and altered synaptic AMPA responses of corresponding hBRaf mutant-expressing CA1 (n=21; normality test, $P=0.30$; constant variance, $P=0.14 ; r=0.812, P<0.001)$ or LA $(n=21$; normality test, $P=0.25$; constant variance, $P=0.77$; $r=$ $0.700, P<0.001 /$ neurons in rats. The three plots at the right show correlations between cognitive impairments of CFC patients and altered amounts of freezing in contextual $(n=21$; normality test, $P=0.70$; constant variance, $P=0.53 ; r=0.923, P<0.001)$ and cued $(n=21$; normality test, $P=0.34$; constant variance, $P=0.96 ; r=0.940, P<0.001$ ) fear conditioning tests and altered amounts of correct choices in $\mathrm{Y}$ maze tests $(n=21$; normality test, $P=0.83$; constant variance, $P=0.92 ; r=0.848, P<0.001$; linear regression $t$-test $)$ in rats. $(B)$ The schematic drawing shows that CFC-and/or cancer-linked mutations in the cysteine-rich domain, G loop, nucleotide-binding pocket (NBP), $\mathrm{C}$ loop, DFG motif, A loop, and C terminus cause a wide range of increases or decreases in BRaf signaling. Note the predominant BRaf signaling deficits caused by a few mutations at sites around the G loop, NBP, C loop, and A loop potentially involved in interaction of ATP, some of which may also affect the turnover of BRaf mutants (cf. Min et al. 2015). 
synapses remains unclear. We show here that BRaf relays Ras activity to MEK-ERK, completing this neuronal signal transduction cascade. Because genetic defects have been identified in almost every signaling molecule in the Ras family GTPase signaling pathways and linked to a large number of cognitive disorders (Costa and Silva 2003; Thomas and Huganir 2004; Stornetta and Zhu 2011), understanding the architecture of the NMDA-RCaMKII-SynGap-Ras-BRaf-MEK-ERK signal transduction cascade should help one to portray the pathogenesis and guide the future development of treatments for these insidious diseases.

Here, we report the development of a fast cost-efficient lentivirus-based gene replacement method that permits large-scale interrogation of genes and their mutations. The method seems to be generally applicable to other genes, since the same biochemical and functional replacements have been achieved with several other molecules sized from $\sim 15$ to $120 \mathrm{KDa}$ (our unpublished data). While lentiviral constructs with proper shRNAs efficiently knocked down these molecules by $70 \%-95 \%$, its generic EF1 a promoter invariably overexpressed recombinant proteins (by 200\%-1200\%). Including an additional IRES sequence added another gene copy of fluorescence proteins and reduced the expression of recombinant proteins to levels comparable $(\sim 50 \%-200 \%)$ with endogenous proteins. Fine-tuning the promoter and/or knockdown efficiencies (i.e., using other promoters, such as CMV, Thy, CaMKII, and Synapsin [Dittgen et al. 2004], and other shRNA sequences) could bring functional expression of recombinant proteins to levels similar to those of endogenous proteins during the 7-d expression period, paving the way for large-scale analysis. As shown in this study, analyzing a large number of mutations can not only provide more rigorous results but also shed light on the general properties and functions of genes of interest.

The lentivirus-based gene replacement method is ideal for defining the signaling defects responsible for a variety of human diseases. Our analysis of $\sim 40$ CFC-linked BRaf mutations provides undisputed evidence supporting the notion that loss and gain of functions of the same signaling molecules lead to the same cognitive phenotypes (Costa and Silva 2003; Thomas and Huganir 2004; Stornetta and Zhu 2011). In particular, our results indicate that loss-of-function and gain-of-function BRaf mutations block and occlude synaptic potentiation, respectively. These findings validate the long-standing hypothesis that both down-regulation and up-regulation of synaptic Ras signaling may force it to deviate from its "happy medium" point and thus shrink its signaling dynamic range (i.e., being either too weak to achieve proper activation or too strong to stay away from saturation at synapses), which reduces the capacity for synaptic plasticity (McCormack et al. 2006). Therefore, the results provide a mechanistic explanation for the observations that loss and gain of functions of synaptic signaling molecules impair cognition (Costa and Silva 2003; Thomas and Huganir 2004; Stornetta and Zhu 2011). The findings may account for the clinical conundrum that off-label medications with FDA-approved Ras signaling modulators fre- quently failed to produce the desired improvement in cognitive performance in patients; at times, the treatments worsen the prognosis, presumably due to modulating Ras signaling in the wrong direction or to the wrong degree. This study also verifies the effectiveness of our new method for building up the scientific foundation necessary for designing effective therapeutic treatments for CFC syndrome because it defines the precise signaling effect of all 40 CFC-linked BRaf mutations with multiple simultaneous patch-clamp recordings that compare synaptic transmission of neighboring neurons in the same animals. With this method, we expect that it should be feasible to carry out large-scale interrogation of many other genes and mutations associated with various mental, neurological, and psychiatric disorders; e.g., 20 sporadic autism spectrum disorder-associated mutations on PTEN, 20 Costello syndrome-associated mutations on Ras, $\sim 60$ Noonan syndrome-associated mutations on PTPN11, 40 schizophrenia-associated mutations on NRG1, and $\sim 50$ frontotemporal dementiaassociated mutations on MAPT (Schubbert et al. 2007; Aoki et al. 2008; Hobert and Eng 2009; Zhou and Parada 2012; Lee et al. 2014; Mei and Nave 2014; Ghetti et al. 2015). Interestingly, among the 20 cancer-linked BRaf mutations examined in this study, about one-third of them actually depressed BRaf signaling (Fig. 8B), suggesting an alternative oncogenesis mechanism presumably involved in the secondary enhancement of CRaf signaling. This idea can be verified by systematically analyzing 350 cancer-linked BRaf mutations in nonneuronal cells with the same gene replacement method. Precisely defining how each of the disease-linked human mutations alters Ras signaling (both the direction and degree) is central to using the right drug at the right dose at the right time to rescue widely varied deficits in synaptic transmission, cognitive behavior, and other medical conditions or developing precision medications for individual patients.

\section{Materials and methods}

\section{Animal preparation}

Totals of 3725 male and female Sprague Dawley rats and 90 male and female C57BL/6 mice were used to prepare cultured neurons, cultured slices, intact brain expression, and behavior experiments in this study. Animals were maintained in the animal facility at the University of Virginia and family- or pair-housed in the temperature-controlled animal room with a 12-h/12-h light/dark cycle. All procedures for animal surgery and maintenance were performed following protocols approved by the Animal Care and Use Committee of the University of Virginia and in accordance with US National Institutes of Health guidelines.

\section{Cultured neuron preparation}

Hippocampal cultured neurons were prepared from rat embryos at embryonic day 18 (E18; $n=15$ pregnant females) as described previously (Zhang and Macara 2006) with minor modifications. Cells were dissociated mechanically after trypsin treatment and plated onto poly-D-lysine-coated (Sigma, P1149) plastic culture 
dishes at a density of $\sim 5 \times 10^{4}$ cells per square centimeter. Plated cells were recovered in the plating medium (DMEM with $2 \mathrm{mM}$ glutamine, $10 \%$ FBS, $0.45 \%$ glucose, $0.11 \mathrm{mg} / \mathrm{mL}$ sodium pyruvate) for 3-4 h and then maintained in the neurobasal medium (Invitrogen, 21103-049) supplemented with B27 (Invitrogen, 17504-044) and glutamax (Invitrogen, 35050-061) before use.

\section{Human neuron preparation}

Human neurons were prepared from H1 ESCs and GM-1 cells using protocols modified from previous studies (Pang et al. 2011; Williams et al. 2014; Du et al. 2015; Yi et al. 2016). Briefly, to prepare hESC-induced neurons, H1 ESCs were cultured feeder-free on Corning Matrigel membrane matrix-coated (Thermo Fisher) plates and fed daily with mTeSR 1 medium (Stem Cell Technologies). Cells were passaged by dissociating the cell cultures into single cells using Accutase (Stem Cell Technologies) and replated in mTeSR 1 medium containing $5 \mu$ M ROCK inhibitor (Stemolecule Y27632, Stemgent) every 4-6 d. To induce neuronal differentiation, ESCs were dissociated, collected, and centrifuged to a pellet; resuspended in a lentiviral cocktail consisting of mTeSR 1, $5 \mu \mathrm{M}$ ROCK inhibitor, and lentiviral-Ngn2 and -rtTA supernatant; and then seeded onto Matrigel-coated six-well plates at a density of $8 \times 10^{5}$ cells per well. The lentiviral cocktail was removed and replaced with neurobasal (ThermoFisher) medium containing Gem21 supplement (Gemini), L-glutamine, and $2 \mu \mathrm{g} / \mathrm{mL}$ doxycycline (MP Biomedicals) $8 \mathrm{~h}$ later. Neuron selection with $1 \mu \mathrm{g} / \mathrm{mL}$ puromycin was performed on days 3-4 after neuronal induction with doxycycline. On day 4.5 , cells were dissociated and replated into 24-well dishes at a density of $2 \times 10^{5}$ (Western blotting) or $1 \times$ $10^{5}$ (electrophysiology) neurons and $1.5 \times 10^{5}$ glia per well in neuronal culture medium containing neurobasal, L-glutamine, Gem21, $2 \mu \mathrm{g} / \mathrm{mL}$ doxycycline, $10 \mathrm{ng} / \mathrm{mL}$ BDNF, $10 \mathrm{ng} / \mathrm{mL}$ GDNF, $10 \mathrm{ng} / \mathrm{mL} \mathrm{NT3}$, and $0.2 \mu \mathrm{g} / \mathrm{mL}$ Laminin-1, and the cultures were half-fed every $2-3 \mathrm{~d}$. AraC was added to the culture medium $48 \mathrm{~h}$ later to inhibit glial overgrowth. On day 31.5, lentiviral replacement constructs harboring hBRaf mutants of G469E, $\mathrm{K} 482 \mathrm{M}, \mathrm{K} 499 \mathrm{E}$, and V600E were added in refreshing culture medium. On day 35, cells were detached with Accutase, centrifuged, washed in DPBS, and then lysed for biochemical analysis.

To prepare human iPSC-derived neurons, GM-1 cells were passaged onto the irradiated mouse embryonic fibroblast plates using $1 \mathrm{mg} / \mathrm{mL}$ dispase and cultured in hESC medium for $7 \mathrm{~d}$ until $\sim 90 \%$ confluence. GM- 1 cells were then differentiated into neuroepithelial progenitors by replacing hESC medium with a chemically defined neural medium (CDM) containing DMEM/F12, neurobasal medium at $1: 1,1 \times \mathrm{N} 2,0.5 \times \mathrm{B} 27,0.5 \times$ glutamax, $1 \times$ NEAA (all from Invitrogen), and an additional $2 \mu \mathrm{M}$ DMH1 (Torcris) and $2 \mu \mathrm{M}$ SB 431542 (Stemgent), which was replaced daily for $8 \mathrm{~d}$. Neuroepithelial progenitors were dissociated with $1 \mathrm{mg} / \mathrm{mL}$ dispase and cultured in the neuronal induction medium (NIM) containing DMEM/F12, $1 \times \mathrm{N} 2$, and $1 \times$ NEAA for $10 \mathrm{~d}$ to induce neuronal progenitors. Neuronal progenitors were dissociated with TrypLE enzyme into single cells at a density of $4 \times 10^{5}$ cells per well, plated on terminally differentiated $\mathrm{H} 9$ astrocytes differentiated from late astroglial progentitors at a density of $8 \times 10^{4}$ cells per well, and fed with the neuronal differentiation medium (NDM) containing neurobasal medium, $1 \times \mathrm{N} 2,1 \times \mathrm{B} 27$, $0.5 \times$ glutamax, $1 \times$ NEAA, $0.1 \mu \mathrm{M}$ compound $\mathrm{E}$ (Calbiochem), and $0.5 \mu \mathrm{M}$ ROCK inhibitor. AraC $(2 \mu \mathrm{M})$ was included in the NDM to inhibit proliferation, and the NDM was refreshed every $4 \mathrm{~d}$. Thirty-five days later, lentiviral replacement constructs harboring hBRaf mutants of G469E, K482M, K499E, and V600E were added in refreshing culture medium. On day 38.5, cells were detached with $0.25 \%$ Trypsin-EDTA (Invitrogen), washed twice in DPBS, and then lysed for biochemical analysis.

\section{Cultured slice preparation}

Cultured slices were prepared from postnatal 6- to 7-d-old rats or mice (postnatal day 6-7 [P6-P7]; $n=1,851$ for rats; $n=30$ for mice) following our previous studies (Lim et al. 2014; Wang et al. 2015a). In brief, the hippocampi were dissected out in ice-cold HEPES-buffered Hanks' solution (pH 7.35) under sterile conditions, sectioned into 400- $\mu \mathrm{m}$ slices on a tissue chopper, and explanted onto a Millicell-CM membrane $(0.4-\mu \mathrm{m}$ pore size; Millipore). The membranes were then placed in $750 \mu \mathrm{L}$ of MEM culture medium containing $30 \mathrm{mM}$ HEPES, $20 \%$ heat-inactivated horse serum, $1.4 \mathrm{mM}$ glutamine, $16.25 \mathrm{mM}$ D-glucose, $5 \mathrm{mM}$ $\mathrm{NaHCO}_{3}, 1 \mathrm{mM} \mathrm{CaCl}, 2 \mathrm{mM} \mathrm{MgSO}, 1 \mathrm{mg} / \mathrm{mL}$ insulin, $0.012 \%$ ascorbic acid (pH 7.28), and $320 \mathrm{mM}$ osmolarity. Cultured slices were maintained at $35^{\circ} \mathrm{C}$ in a humidified incubator (ambient air enriched with $5 \% \mathrm{CO}_{2}$ ).

\section{Constructs of recombinant proteins and expression}

The human BRaf(WT) cDNA (Open Biosystems, clone ID 8327547; accession no. BC112079) was subcloned into pEGFPC1 (enhanced GFP; Clontech Laboratories) to produce GFPhBRaf, which was then used as the template for GFP-hBRaf(CA) $(\mathrm{T} 599 \rightarrow \mathrm{E} / \mathrm{S} 602 \rightarrow \mathrm{D})$ (Zhang and Guan 2000), GFP-hBRaf(DN) $(\mathrm{K} 482 \rightarrow \mathrm{M})$, and various GFP-tagged CFC- and/or cancer-linked hBRaf mutant constructs using QuikChange XL site-directed mutagenesis kit (Stratagene). GFP-BRaf was subsequently subcloned in Sindbis viral vector. To generate lentiviral replacement constructs, a palmitoylation signal sequence of GAP43 (Pal) was fused to the $\mathrm{N}$ terminus of GFP to generate PalGFP. PalGFP and GFP-hBRaf were subcloned before and after an IRES sequence of the pCITE vector to produce PalGFP-IRES-GFP-hBRaf. PalGFPIRES-GFP-hBRaf and rat BRaf targeting or shRNA or S-shRNA were then subcloned into lentiviral vector pLVTHM after EF1a and $\mathrm{H} 1$ promoters, respectively, to generate (S-)shRNA-PalGFPIRES-GFP-hBRaf. In some experiments, GFP was replaced with mCherry or CFP (gifts from Dr. Roger Tsien) in the construct. The recombinant hBRaf expression efficiencies of several promoters (e.g., CMV, Thy, CaMKII, and Synapsin) and knockdown efficiencies of four shRNAs that target different BRaf sequences were assessed. We found that combination of EFla and one shRNA produced the most rapid and equivalent replacement. Thus, the EF1a, shRNA, and its control scrambled construct were chosen for use in this study.

The intellectual impairment of CFC syndrome first appears in $\sim 1.5$ - to 2.5-yr-old children, who are developmentally equivalent to $\sim 2$ - to 4 -wk-old rodents. This matches the timing when CaMKII-Ras signaling starts to functionally replace the neonatal PKA signaling and take control of synaptic plasticity (Yasuda et al. 2003; Stornetta and Zhu 2011). Thus, for in vitro expression, CA1 pyramidal neurons in hippocampal cultured slices were infected after $8-18 \mathrm{~d}$ in vitro with lentivirus or Sindbis virus or transfected with a Bio-Rad Helios gene gun and then incubated on culture medium and $5 \% \mathrm{CO}_{2}$ before experiments. For in vivo expression, P18-P28 rats were initially anesthetized by an intraperitoneal injection of $10 \mathrm{mg} / \mathrm{kg}$ ketamine and $2 \mathrm{mg} / \mathrm{kg}$ xylazine. Animals were then placed in a stereotaxic frame, and one or multiple $\sim 1 \times 1 \mathrm{~mm}$ holes were opened above the right or both sides of the somatosensory cortex. A glass pipette was used to make pressure injections of lentiviral solution in the hippocampal CA1 region or LA according to stereotaxic coordinates. For electrophysiology recordings, one injection of $\sim 100 \mathrm{~nL}$ of diluted viral solution was made into the right hippocampus or amygdala to create sparse expression in neurons in 314 animals. For behavioral tests, multiple injections of $\sim 6 \mu \mathrm{L}$ of viral solution were delivered along the entire hippocampal CA1 (10-12 injections) or 
LA (two injections) of both sides to achieve massive expression in about one third of CA1 $(34.1 \pm 0.7 \% ; n=53)$ or LA $(37.7 \pm 1.0 \%$; $n$ $=44$ ) neurons in 1483 animals (Figs. 6A, 7A). After injection, animals were allowed to recover from the anesthesia and returned to their cages. Experiments were typically performed within $18 \mathrm{~h} \pm 2$ $\mathrm{h}$ after Sindbis viral infection and biolistic transfection or within 2-7 d after lentiviral infection unless stated otherwise.

\section{Biochemical analysis}

Hippocampal extracts were prepared by homogenizing hippocampal CAl regions isolated from cultured rat or mouse hippocampal slices (Zhu et al. 2002; Qin et al. 2005). Human neuronal extracts were prepared by homogenizing ESC-induced or iPSC-derived neuron cultures. Membranes were blotted with anti-BRaf (1:1000; BD Transduction Laboratories) or anti-phospho-ERK (1:10,000; Cell Signaling Technology), stripped, and reblotted with anti-ERK (1:1000; Cell Signaling). Western blots were quantified by chemiluminescence and densitometric scanning of the films under linear exposure conditions.

\section{Electrophysiology}

Simultaneous multiple whole-cell recordings were obtained from nearby infected/transfected and noninfected/nontransfected CA1 pyramidal neurons or LA large pyramidal-like neurons under visual guidance with fluorescence and transmitted light illumination (Lim et al. 2014; Wang et al. 2015b) using up to four Axopatch-200B or Axoclamp 2B amplifiers (Axon Instruments). Bath solution $\left(29^{\circ} \mathrm{C} \pm 1.5^{\circ} \mathrm{C}\right)$, unless otherwise stated, contained 119 $\mathrm{mM} \mathrm{NaCl}, 2.5 \mathrm{mM} \mathrm{KCl}, 4 \mathrm{mM} \mathrm{CaCl} 2,4 \mathrm{mM} \mathrm{MgCl} 2,26 \mathrm{mM}$ $\mathrm{NaHCO}_{3}, 1 \mathrm{mM} \mathrm{NaH} \mathrm{PO}_{4}, 11 \mathrm{mM}$ glucose, $0.1 \mathrm{mM}$ picrotoxin (PTX), and $0.002 \mathrm{mM} 2$-chloroadenosine (pH 7.4) and was gassed with $5 \% \mathrm{CO}_{2} / 95 \% \mathrm{O}_{2}$. 2-chloroadenosine was included to prevent bursting, and PTX was excluded when GABA responses were examined. For experiments in which slices were maintained in culture with an additional $10 \mathrm{mM} \mathrm{Mg}^{2+}$ (to suppress synaptic activity), $100 \mu \mathrm{M}$ DL-APV, $25 \mu \mathrm{M}$ PD98059, $10 \mu \mathrm{M}$ U0126, or 5 $\mu \mathrm{g} / \mathrm{mL} \alpha$-BP7 (Abcam), these reagents were included at the time of $\mathrm{Mg}^{2+}$, DL-APV, PD98059, and U0126 $1.5 \mathrm{~d}$ (PD98059) or $3.5 \mathrm{~d}$ (a-BP7) after viral infection and removed during recordings. Patch recording pipettes (3-6 $\mathrm{M} \Omega$ ) for current (voltage-clamp) recordings contained $115 \mathrm{mM}$ cesium methanesulfonate, $20 \mathrm{mM}$ CsCl, 10 mM HEPES, $2.5 \mathrm{mM} \mathrm{MgCl}_{2}, 4 \mathrm{mM} \mathrm{Na}_{2} \mathrm{ATP}, 0.4 \mathrm{mM}$ $\mathrm{Na}_{3} \mathrm{GTP}, 10 \mathrm{mM}$ sodium phosphocreatine, $0.6 \mathrm{mM}$ EGTA, and $0.1 \mathrm{mM}$ spermine (at $\mathrm{pH} 7.25$ ), and those for voltage (currentclamp) recordings contained $115 \mathrm{mM}$ potassium gluconate, $10 \mathrm{mM}$ HEPES, $2 \mathrm{mM} \mathrm{MgCl}$, $2 \mathrm{mM} \mathrm{MgATP}, 2 \mathrm{mM} \mathrm{Na} \mathrm{m}_{2} \mathrm{ATP}$, $0.3 \mathrm{mM} \mathrm{Na}_{3} \mathrm{GTP}$, and $20 \mathrm{mM} \mathrm{KCl}$ (pH 7.25). Synaptic responses were evoked by bipolar electrodes with single voltage pulses $(200 \mu s e c$, up to $20 \mathrm{~V}$ ) placed in the stratum radiatum $\sim 300-500$ $\mu \mathrm{m}$ from CA1 neurons or in the ventral striatum $\sim 200-400 \mu \mathrm{m}$ from LA neurons. Synaptic AMPA and NMDA responses at -60 $\mathrm{mV}$ and $+40 \mathrm{mV}$ were averaged over 90 trials. To minimize the effect from AMPA responses, the peak NMDA responses at $+40 \mathrm{mV}$ were measured after digital subtraction of the estimated AMPA responses at $+40 \mathrm{mV}$.

\section{Behavioral analysis}

Fear conditioning tests and Y-maze tests, started at approximately 5:00 pm, followed the previous reports (Schmitt et al. 2005; Lim et al. 2014). Briefly, fear conditioning was performed in an isolated shock chamber (Coulbourn Instruments). The fear condition- ing training consisted of a 3-min exposure of rats to the conditioning box (context) followed by a foot shock ( 2 sec at 0.8 $\mathrm{mA}$ ) after a preceding tone ( $30 \mathrm{sec}$ at $3 \mathrm{kHz}$ and $75 \mathrm{~dB}$ ). The memory test was performed $24 \mathrm{~h}$ later by re-exposing the animals for 5 min to the conditioning context and, $1 \mathrm{~h}$ later, to a novel context for $3 \mathrm{~min}$ followed by a tone ( $3 \mathrm{~min}$ at $3 \mathrm{kHz}$ and $75 \mathrm{~dB}$ ). All data were recorded using a video-based FreezeFrame system (Coulbourn Instruments). Freezing, defined as a lack of movement except for heartbeat and respiration associated with a crouching posture, and locomotor activity were analyzed offline with the FreezeFrame software (Coulbourn Instruments).

Y-maze learning tests were carried out using an elevated Ymaze with one start arm and two goal arms $(56.5 \times 12 \mathrm{~cm}$ each) surrounded by an opaque 26-cm-high wall (San Diego Instruments). A food well, located $5 \mathrm{~cm}$ from the end of each goal arm, was filled with $0.1 \mathrm{~mL}$ of sucrose-sweetened nonfat dry milk $(12.5 \%)$ as a reward. Animals were initially habituated to the Y-maze and milk reward. Once all of the animals were running freely on the Y-maze and readily consuming the milk rewards, testing began. Animals were trained and tested on a conditional learning task, in which interchangeable floor inserts, either plain white or black wire mesh, covering the whole of the start arm, were used as a conditional cue. The presence of the plain white insert in the start arm indicated that the $0.1-\mathrm{mL}$ milk reward was available in the right goal arm, whereas the presence of the black wire mesh insert was associated with the reward in the left arm. Animals received 14 training-testing sessions consisting of 10 trials per session with an ITI of $5 \mathrm{~min}$. Each session consisted of five trials with each of the two floor inserts, and no more than three consecutive trials with the same floor insert, according to a pseudorandom sequence. The percentage of correct responses-or entering the floor-matching goal arm and consuming the milk reward-was calculated for each session.

\section{Correlation analysis with the clinical data}

Although variance exists due to medical, environmental, and other genetic factors, CFC patients carrying the same BRaf mutations exhibit roughly similar intellectual disabilities (see Supplemental Table S28; Yoon et al. 2007; Demir et al. 2010; Pierpont et al. 2010; Alfieri et al. 2014). We averaged the published cognitive measurements of CFC patients, arbitrarily converting the mild, moderate, severe, and profound mental impairments into the scores of $0.5,1.5,2.5$, and 3.5, respectively (Supplemental Table S28). Next, we compared the average cognitive impairment scores of human patients with the alterations in synaptic transmission in hBRaf mutant-expressing neurons or deficits in learning in hBRaf mutant-expressing animals.

\section{Statistical analysis}

Statistical results were reported as mean \pm SEM. Animals or cells were randomly assigned into control or experimental groups, and investigators were blinded to experiment treatments in cultured slices and animals. Given the negative correlation between the variation and square root of sample number $(n)$, the group sample size was typically set to be $\sim 16-36$ to optimize the efficiency and power of statistical tests. The statistical significances of the means $(P \leq 0.05$; two sides) were determined using Wilcoxon and Mann-Whitney rank sum nonparametric tests for paired and unpaired samples, respectively, and statistical significances of the linear relationships of two data groups were determined using linear regression $t$-tests, which provided the normality and constant variance tests that were passed. 


\section{Acknowledgments}

We thank members of the Zhu laboratory for comments and technical assistance, and Dr. Ian Macara, Dr. Tom Südhof, and Dr. Roger Tsien for reagents and/or use of laboratory resources. This study is supported in part by National Natural Science Foundation of China grants NSFC30728012 to S.W. and J.J.Z., and NSFC81625006 and NSFC31471024 to Y.S.; Chinese Ministry of Education Project 111 Program grant B13026 to Y.S. and J.J.Z.; State Major Research and Development Program of China grant 2016YFA0500400 to S.W. and Y.S.; the Japan Society for the Promotion of Science (Y.A.); the Robert Wood Johnson Foundation (Z.P.P.); National Honor Scientist Program of Korea grants NRF2012R1A3A1050385 to B.-K.K., and NRF2016R1D1A1 B03931525 to C.-S.L.; the Howard Hughes Medical Institute (R.L.H.); and National Institute of Health grants MH108321 to V.M., NS065183 and NS089578 to H.Z., HD064743 to Q.C., AA023797 to Z.P.P., MH64856 and NS036715 to R.L.H., and NS053570, NS091452, and NS092548 to J.J.Z.

\section{References}

Alfieri P, Piccini G, Caciolo C, Perrino F, Gambardella ML, Mallardi M, Cesarini L, Leoni C, Leone D, Fossati C, et al. 2014. Behavioral profile in RASopathies. Am J Med Genet A 164A: 934-942.

Aoki Y, Niihori T, Narumi Y, Kure S, Matsubara Y. 2008. The RAS/MAPK syndromes: novel roles of the RAS pathway in human genetic disorders. Hum Mutat 29: 992-1006.

Araki Y, Zeng M, Zhang M, Huganir RL. 2015. Rapid dispersion of SynGAP from synaptic spines triggers AMPA receptor insertion and spine enlargement during LTP. Neuron 85: 173-189.

Caunt CJ, Sale MJ, Smith PD, Cook SJ. 2015. MEK1 and MEK2 inhibitors and cancer therapy: the long and winding road. Nat Rev Cancer 15: 577-592.

Chen AP, Ohno M, Giese KP, Kuhn R, Chen RL, Silva AJ. 2006. Forebrain-specific knockout of B-raf kinase leads to deficits in hippocampal long-term potentiation, learning, and memory. J Neurosci Res 83: 28-38.

Costa RM, Silva AJ. 2003. Mouse models of neurofibromatosis type I: bridging the GAP. Trends Mol Med 9: 19-23.

Demir E, Mancano G, Pomponi MG, Ozcelik A, Gucuyener K, Neri G. 2010. Cardio-facio-cutaneous syndrome: phenotypic variability and differential diagnosis in 3 cases with de novo BRAF mutations. Neuropediatrics 41: 127-131.

Dittgen T, Nimmerjahn A, Komai S, Licznerski P, Waters J, Margrie TW, Helmchen F, Denk W, Brecht M, Osten P. 2004. Lentivirus-based genetic manipulations of cortical neurons and their optical and electrophysiological monitoring in vivo. Proc Natl Acad Sci 101: 18206-18211.

Du ZW, Chen H, Liu H, Lu J, Qian K, Huang CL, Zhong X, Fan F, Zhang SC. 2015. Generation and expansion of highly pure motor neuron progenitors from human pluripotent stem cells. Nat Commun 6: 6626.

English JD, Sweatt JD. 1997. A requirement for the mitogen-activated protein kinase cascade in hippocampal long term potentiation. J Biol Chem 272: 19103-19106.

Ghetti B, Oblak AL, Boeve BF, Johnson KA, Dickerson BC, Goedert M. 2015. Invited review: frontotemporal dementia caused by microtubule-associated protein tau gene (MAPT) mutations: a chameleon for neuropathology and neuroimaging. Neuropathol Appl Neurobiol 41: 24-46.

Govek EE, Newey SE, Van Aelst L. 2005. The role of the Rho GTPases in neuronal development. Genes Dev 19: 1-49.
Hobert JA, Eng C. 2009. PTEN hamartoma tumor syndrome: an overview. Genet Med 11: 687-694.

Holderfield M, Deuker MM, McCormick F, McMahon M. 2014. Targeting RAF kinases for cancer therapy: BRAF-mutated melanoma and beyond. Nat Rev Cancer 14: 455-467.

Husi H, Ward MA, Choudhary JS, Blackstock WP, Grant SG. 2000. Proteomic analysis of NMDA receptor-adhesion protein signaling complexes. Nat Neurosci 3: 661-669.

Kielland A, Bochorishvili G, Corson J, Zhang L, Rosin DL, Heggelund P, Zhu JJ. 2009. Activity patterns govern synapse-specific AMPA-R trafficking between deliverable and synaptic pools. Neuron 62: 84-101.

Kim MJ, Dunah AW, Wang YT, Sheng M. 2005. Differential roles of NR2A- and NR2B-containing NMDA receptors in Ras-ERK signaling and AMPA receptor trafficking. Neuron 46: 745-760.

Lee SI, Escobedo-Lozoya Y, Szatmari EM, Yasuda R. 2009. Activation of CaMKII in single dendritic spines during long-term potentiation. Nature 458: 299-304.

Lee YS, Ehninger D, Zhou M, Oh JY, Kang M, Kwak C, Ryu HH, Butz D, Araki T, Cai Y, et al. 2014. Mechanism and treatment for learning and memory deficits in mouse models of Noonan syndrome. Nat Neurosci 17: 1736-1743.

Li Z, Jo J, Jia JM, Lo SC, Whitcomb DJ, Jiao S, Cho K, Sheng M. 2010. Caspase-3 activation via mitochondria is required for long-term depression and AMPA receptor internalization. Cell 141: 859-871.

Lim CS, Hoang ET, Viar KE, Stornetta RL, Scott MM, Zhu JJ. 2014. Pharmacological rescue of Ras signaling, GluA1-dependent synaptic plasticity, and learning deficits in a fragile $\mathrm{X}$ model. Genes Dev 28: 273-289.

Man HY, Wang Q, Lu WY, Ju W, Ahmadian G, Liu L, D'Souza S, Wong TP, Taghibiglou C, Lu J, et al. 2003. Activation of PI3kinase is required for AMPA receptor insertion during LTP of mEPSCs in cultured hippocampal neurons. Neuron 38: 611-624.

McCormack SG, Stornetta RL, Zhu JJ. 2006. Synaptic AMPA receptor exchange maintains bidirectional plasticity. Neuron 50: $75-88$.

Mei L, Nave KA. 2014. Neuregulin-ERBB signaling in the nervous system and neuropsychiatric diseases. Neuron 83: 27-49.

Min SW, Chen X, Tracy TE, Li Y, Zhou Y, Wang C, Shirakawa K, Minami SS, Defensor E, Mok SA, et al. 2015. Critical role of acetylation in tau-mediated neurodegeneration and cognitive deficits. Nat Med 21: 1154-1162.

Morice C, Nothias F, Konig S, Vernier P, Baccarini M, Vincent JD, Barnier JV. 1999. Raf-1 and B-Raf proteins have similar regional distributions but differential subcellular localization in adult rat brain. Eur J Neurosci 11: 1995-2006.

Nishiyama J, Yasuda R. 2015. Biochemical computation for spine ctructural plasticity. Neuron 87: 63-75.

Ozkan ED, Creson TK, Kramar EA, Rojas C, Seese RR, Babyan AH, Shi Y, Lucero R, Xu X, Noebels JL, et al. 2014. Reduced cognition in Syngapl mutants is caused by isolated damage within developing forebrain excitatory neurons. Neuron 82: 1317-1333.

Pang ZP, Yang N, Vierbuchen T, Ostermeier A, Fuentes DR, Yang TQ, Citri A, Sebastiano V, Marro S, Sudhof TC, et al. 2011. Induction of human neuronal cells by defined transcription factors. Nature 476: 220-223.

Patterson MA, Szatmari EM, Yasuda R. 2010. AMPA receptors are exocytosed in stimulated spines and adjacent dendrites in a Ras-ERK-dependent manner during long-term potentiation. Proc Natl Acad Sci 107: 15951-15956. 
Pavlowsky A, Chelly J, Billuart P. 2012. Emerging major synaptic signaling pathways involved in intellectual disability. Mol Psychiatry 17: 682-693.

Pierpont EI, Pierpont ME, Mendelsohn NJ, Roberts AE, TworogDube E, Rauen KA, Seidenberg MS. 2010. Effects of germline mutations in the Ras/MAPK signaling pathway on adaptive behavior: cardiofaciocutaneous syndrome and Noonan syndrome. Am J Med Genet A 152A: 591-600.

Qin Y, Zhu Y, Baumgart JP, Stornetta RL, Seidenman K, Mack V, van Aelst L, Zhu JJ. 2005. State-dependent Ras signaling and AMPA receptor trafficking. Genes Development 19: 2000-2015.

Rauen KA. 2013. The RASopathies. Annu Rev Genomics Hum Genet 14: 355-369.

Samatar AA, Poulikakos PI. 2014. Targeting RAS-ERK signalling in cancer: promises and challenges. Nat Rev Drug Discov 13: 928-942.

Schmitt WB, Sprengel R, Mack V, Draft RW, Seeburg PH, Deacon RM, Rawlins JN, Bannerman DM. 2005. Restoration of spatial working memory by genetic rescue of GluR-A-deficient mice. Nat Neurosci 8: 270-272.

Schubbert S, Shannon K, Bollag G. 2007. Hyperactive Ras in developmental disorders and cancer. Nat Rev Cancer 7: 295-308.

Sheng Y, Zhang L, Su SC, Tsai LH, Zhu JJ. 2016. Cdk5 is a new rapid synaptic homeostasis regulator capable of initiating the early alzheimer-like pathology. Cereb Cortex 26: $2937-2951$.

Siegert S, Seo J, Kwon EJ, Rudenko A, Cho S, Wang W, Flood Z, Martorell AJ, Ericsson M, Mungenast AE, et al. 2015. The schizophrenia risk gene product miR-137 alters presynaptic plasticity. Nat Neurosci 18: 1008-1016.

Stornetta RL, Zhu JJ. 2011. Ras and Rap signaling in synaptic plasticity and mental disorders. Neuroscientist 17: 54-78.

Takahashi T, Svoboda K, Malinow R. 2003. Experience strengthening transmission by driving AMPA receptors into synapses. Science 299: 1585-1588.

Thomas GM, Huganir RL. 2004. MAPK cascade signalling and synaptic plasticity. Nat Rev Neurosci 5: 173-183.

Volk L, Chiu SL, Sharma K, Huganir RL. 2015. Glutamate synapses in human cognitive disorders. Annu Rev Neurosci 38: 127-149.

Wajapeyee N, Serra RW, Zhu X, Mahalingam M, Green MR. 2008. Oncogenic BRAF induces senescence and apoptosis through pathways mediated by the secreted protein IGFBP7. Cell 132: 363-374.

Wang G, Bochorishvili G, Chen Y, Salvati KA, Zhang P, Dubel SJ, Perez-Reyes E, Snutch TP, Stornetta RL, Deisseroth K, et al. 2015a. CaV3.2 calcium channels control NMDA receptor-me- diated transmission: a new mechanism for absence epilepsy. Genes Dev 29: 1535-1551.

Wang G, Wyskiel DR, Yang W, Wang Y, Milbern LC, Lalanne T, Jiang X, Shen Y, Sun Q-Q, Zhu JJ. 2015b. An optogenetics- and imaging-assisted simultaneous multiple patch-clamp recordings system for decoding complex neural circuits. Nat Protoc 10: $397-412$.

Williams EC, Zhong X, Mohamed A, Li R, Liu Y, Dong Q, Ananiev GE, Mok JC, Lin BR, Lu J, et al. 2014. Mutant astrocytes differentiated from Rett syndrome patients-specific iPSCs have adverse effects on wild-type neurons. Hum Mol Genet 23: $2968-2980$.

Yasuda H, Barth AL, Stellwagen D, Malenka RC. 2003. A developmental switch in the signaling cascades for LTP induction. Nat Neurosci 6: 15-16.

Yasuda R, Harvey CD, Zhong H, Sobczyk A, van Aelst L, Svoboda K. 2006. Supersensitive Ras activation in dendrites and spines revealed by two-photon fluorescence lifetime imaging. Nat Neurosci 9: 283-291.

Yi F, Danko T, Botelho SC, Patzke C, Pak C, Wernig M, Sudhof TC. 2016. Autism-associated SHANK3 haploinsufficiency causes Ih channelopathy in human neurons. Science 352: aaf2669.

Yoon G, Rosenberg J, Blaser S, Rauen KA. 2007. Neurological complications of cardio-facio-cutaneous syndrome. Dev Med Child Neurol 49: 894-899.

Zeng M, Shang Y, Araki Y, Guo T, Huganir RL, Zhang M. 2016. Phase transition in postsynaptic densities underlies hormation of synaptic complexes and synaptic plasticity. Cell 166: 1163-1175.e12.

Zhang BH, Guan KL. 2000. Activation of B-Raf kinase requires phosphorylation of the conserved residues Thr598 and Ser601. EMBO I 19: 5429-5439.

Zhang H, Macara IG. 2006. The polarity protein PAR-3 and TIAM1 cooperate in dendritic spine morphogenesis. Nat Cell Biol 8: 227-237.

Zhou J, Parada LF. 2012. PTEN signaling in autism spectrum disorders. Curr Opin Neurobiol 22: 873-879.

Zhu JJ, Esteban JA, Hayashi Y, Malinow R. 2000. Postnatal synaptic potentiation: delivery of GluR4-containing AMPA receptors by spontaneous activity. Nat Neurosci 3: 1098-1106.

Zhu JJ, Qin Y, Zhao M, Van Aelst L, Malinow R. 2002. Ras and Rap control AMPA receptor trafficking during synaptic plasticity. Cell 110: 443-455.

Zhu Y, Pak D, Qin Y, McCormack SG, Kim MJ, Baumgart JP, Velamoor V, Auberson YP, Osten P, van Aelst L, et al. 2005. Rap2-JNK removes synaptic AMPA receptors during depotentiation. Neuron 46: 905-916. 


\section{ERRATUM}

Genes \& Development 31: 537-552 (2017)

\section{Erratum: BRaf signaling principles unveiled by large-scale human mutation analysis with a rapid lentivirus-based gene replacement method}

Chae-Seok Lim, Xi Kang, Vincent Mirabella, Huaye Zhang, Qian Bu, Yoichi Araki, Elizabeth T. Hoang, Shiqiang Wang, Ying Shen, Sukwoo Choi, Bong-Kiun Kaang, Qiang Chang, Zhiping P. Pang, Richard L. Huganir, and J. Julius Zhu

In the above-mentioned article, the abstract and first paragraph contained two typographical errors that have been corrected in both the PDF and full-text HTML files online. The phrase "small GTPase" was deleted, and "gene mutation-cell behavior" should read "gene-mutation-cell-behavior." The errors have been corrected in both the PDF and full-text HTML files online.

doi: 10.1101/gad.300863.117 


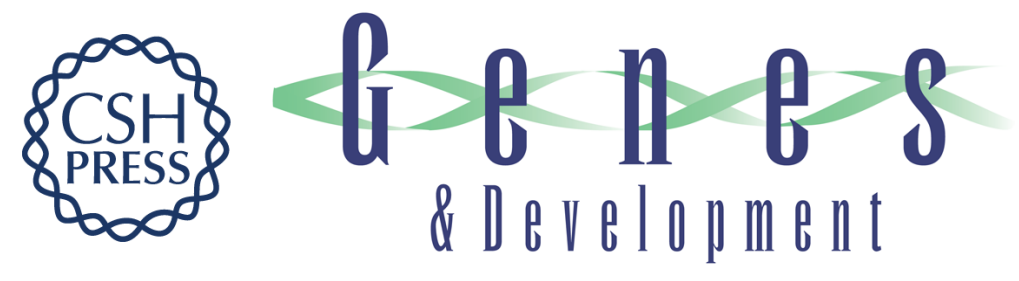

\section{BRaf signaling principles unveiled by large-scale human mutation analysis with a rapid lentivirus-based gene replacement method}

Chae-Seok Lim, Xi Kang, Vincent Mirabella, et al.

Genes Dev. 2017, 31:

Access the most recent version at doi:10.1101/gad.294413.116

\section{Supplemental http://genesdev.cshlp.org/content/suppl/2017/04/12/31.6.537.DC1 Material}

Related Content

Erratum: BRaf signaling principles unveiled by large-scale human mutation analysis with a rapid lentivirus-based gene replacement method Chae-Seok Lim, Xi Kang, Vincent Mirabella, et al.

Genes Dev. April , 2017 31: 846

References

This article cites 58 articles, 10 of which can be accessed free at: http://genesdev.cshlp.org/content/31/6/537.full.html\#ref-list-1

Articles cited in: http://genesdev.cshlp.org/content/31/6/537.full.htmI\#related-urls

Creative This article is distributed exclusively by Cold Spring Harbor Laboratory Press for the first Commons six months after the full-issue publication date (see

License http://genesdev.cshlp.org/site/misc/terms.xhtml). After six months, it is available under a Creative Commons License (Attribution-NonCommercial 4.0 International), as described at http://creativecommons.org/licenses/by-nc/4.0/.

Email Alerting Receive free email alerts when new articles cite this article - sign up in the box at the top Service right corner of the article or click here.

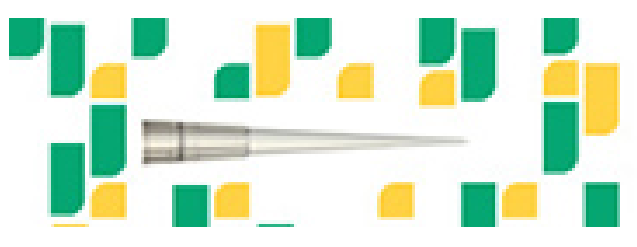

Focused on your science. 